TRANSACTIONS OF THE

AMERICAN MATHEMATICAL SOCIETY

Volume 358, Number 11, November 2006, Pages 4689-4720

S 0002-9947(05)03801-8

Article electronically published on November 1, 2005

\title{
INSEPARABLE EXTENSIONS OF ALGEBRAS OVER THE STEENROD ALGEBRA WITH APPLICATIONS TO MODULAR INVARIANT THEORY OF FINITE GROUPS
}

\author{
MARA D. NEUSEL
}

Dedicated to Clarence W. Wilkerson on the occasion of his 60 th birthday

\begin{abstract}
We consider purely inseparable extensions $\mathrm{H} \hookrightarrow \sqrt[\mathcal{P}^{*}]{\mathrm{H}}$ of unstable Noetherian integral domains over the Steenrod algebra. It turns out that there exists a finite group $G \leq \mathrm{GL}(V)$ and a vector space decomposition $V=W_{0} \oplus W_{1} \oplus \cdots \oplus W_{e}$ such that $\overline{\mathrm{H}}=\left(\mathbb{F}\left[W_{0}\right] \otimes \mathbb{F}\left[W_{1}\right]^{p} \otimes \cdots \otimes \mathbb{F}\left[W_{e}\right]^{p^{e}}\right)^{G}$ and $\sqrt[\mathcal{P}^{*}]{\mathrm{H}}=\mathbb{F}[V]^{G}$, where $\overline{(-)}$ denotes the integral closure. Moreover, $\mathrm{H}$ is Cohen-Macaulay if and only if $\sqrt[\mathcal{P}^{*}]{\mathrm{H}}$ is Cohen-Macaulay. Furthermore, $\overline{\mathrm{H}}$ is polynomial if and only if $\sqrt[\mathcal{P} *]{\mathrm{H}}$ is polynomial, and $\sqrt[\mathcal{P}^{*}]{\mathrm{H}}=\mathbb{F}\left[h_{1}, \ldots, h_{n}\right]$ if and only if

$$
\mathrm{H}=\mathbb{F}\left[h_{1}, \ldots, h_{n_{0}}, h_{n_{0}+1}^{p}, \ldots, h_{n_{1}}^{p}, h_{n_{1}+1}^{p^{2}}, \ldots, h_{n_{e}}^{p^{e}}\right],
$$
\end{abstract}

where $n_{e}=n$ and $n_{i}=\operatorname{dim}_{\mathbb{F}}\left(W_{0} \oplus \cdots \oplus W_{i}\right)$.

\section{INTRODUCTION AND OUTLINE}

Let $\mathbb{K} \hookrightarrow \mathbb{L}$ be an algebraic extension of graded fields. Assume that the smaller field, $\mathbb{K}$, carries an action of the Steenrod algebra $\mathcal{P}^{*}$ of reduced powers. If the extension $\mathbb{K} \hookrightarrow \mathbb{L}$ is separable, then the action of $\mathcal{P}^{*}$ can be uniquely extended to $\mathbb{L}$. In other words, the separable closure of $\mathbb{K}$ as a field over the Steenrod algebra coincides with the separable closure in the category of graded fields; see Proposition 2.2.2 in [3] and Proposition 2.2 in [7].

If the extension, however, is purely inseparable the situation is more delicate: Let $p(X) \in \mathbb{K}[X]$ be the minimal polynomial of $l \in \mathbb{L}$. Since the extension is purely inseparable, we have that

$$
p(X)=X^{p^{e}}-\kappa,
$$

so that $l^{p^{e}}=\kappa$ for some $\kappa \in \mathbb{K}$. Of course, since our fields are graded, we obtain the following condition on the degrees:

$$
\operatorname{deg}(l) p^{e}=\operatorname{deg}(\kappa)
$$

Received by the editors September 18, 2003 and, in revised form, June 22, 2004.

2000 Mathematics Subject Classification. Primary 55S10, 13A50, 13-xx, 55-xx.

Key words and phrases. Inseparable extensions, inseparable closure, Cohen-Macaulay, $\Delta$ relation, $\Delta_{s}$-relation, derivation, restricted Lie algebra, Steenrod algebra, Dickson algebra, invariant rings of finite groups. 
However, the crucial issue is the following. If there were an extension of the $\mathcal{P}^{*}$ action to the larger field $\mathbb{L}$, then

$$
\mathscr{P}^{\Delta_{i}}(\kappa)=0 \quad \forall i
$$

because by equation (囸) the element $\kappa$ is a $p$ th power. Thus, we need to define

$$
\left(\mathscr{P}^{i}(l)\right)^{p^{e}}=\mathscr{P}^{i p^{e}}(\kappa) \in \mathbb{K} \text {. }
$$

The problem is that it does not follow that $\mathscr{P}^{i}(l) \in \mathbb{L}$. Nevertheless, as equation (因) shows, the inseparable closures of $\mathbb{K}$ as a graded field and as a field over the Steenrod algebra coincide. We denote this object by $\sqrt[\mathcal{P}^{*}]{\mathbb{K}}$.

This leads to the following question: Under which conditions can we extend the action of $\mathcal{P}^{*}$ from $\mathbb{K}$ to $\mathbb{L}$ ? Or equivalently, which intermediate fields $\mathbb{K} \subseteq \mathbb{L} \subseteq \sqrt[\mathcal{P}^{*}]{\mathbb{K}}$ are objects in the category of fields over the Steenrod algebra?

In this paper we study these questions in the more general framework of Noetherian integral domains $\mathrm{H}$ over the Steenrod algebra.

In Section 2 we recall the construction of inseparable closures over the Steenrod algebra and its basic properties. To this list we add a few more that will be of use later.

In Sections 3 and 4 we start with the investigation of inseparable extensions $\mathrm{H} \hookrightarrow \sqrt[\mathcal{P}^{*}]{\mathrm{H}}$, where $\sqrt[\mathcal{P}^{*}]{\mathrm{H}}$ is either the symmetric algebra, $\mathbb{F}[V]$, on $V^{*}$ with $V=\mathbb{F}^{n}$, or its field of fractions, $\mathbb{F}(V)$. This has two reasons: for one, $\mathbb{F}(V)$ and $\mathbb{F}[V]$ are universal, in the sense that they are algebraically closed in our category. On the other hand, any unstable Noetherian integral domain $\mathrm{H}$ can be embedded into $\mathbb{F}[V]$ such that the inclusion

$$
\mathrm{H} \hookrightarrow \mathbb{F}[V]
$$

is finite; see the Embedding Theorem, Corollary 6.1.5 in [3]. Thus in Sections 3 and 4 we consider the diagram

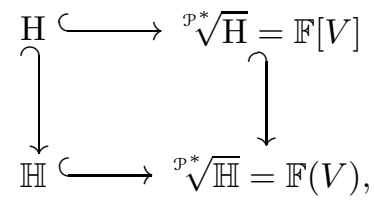

where $\mathbb{H}=F F(\mathrm{H})$ is the field of fractions of $\mathrm{H}$. In Section 3 we treat the case of purely inseparable extensions of exponent one, in Section 4 we look at extensions with higher exponents $e$. Denote by $\overline{(-)}$ the integral closure. The results of these parts show that 1

$$
\begin{aligned}
& \overline{\mathrm{H}}=\mathbb{F}\left[W_{0}\right] \otimes \mathbb{F}\left[W_{1}\right]^{p} \otimes \cdots \otimes \mathbb{F}\left[W_{e}\right]^{p^{e}} \text { and } \\
& \mathbb{H}=\mathbb{F}\left(W_{0}\right) \otimes \mathbb{F}\left(W_{1}\right)^{p} \otimes \cdots \otimes \mathbb{F}\left(W_{e}\right)^{p^{e}}
\end{aligned}
$$

for some vector space decomposition $V=W_{0} \oplus W_{1} \oplus \cdots \oplus W_{e}$; see Theorem 4.13 and Corollary 4.142 $\left.\right|^{2}$ This reproves results in [8], Theorem II, and 3], Theorem 7.2.2. However, the proof presented here has the advantage that it gives precise information on the vector space dimensions of the $W_{i}$ 's.

\footnotetext{
${ }^{1}$ All tensor products in this manuscript are tensor products over the ground field $\mathbb{F}$.

${ }^{2}$ We denote by $\mathbb{F}[V]^{p}$ the algebra $\mathbb{F}\left[x_{1}^{p}, \ldots, x_{n}^{p}\right]$ for $\mathbb{F}[V]=\mathbb{F}\left[x_{1}, \ldots, x_{n}\right]$.
} 
In Section 5 we come to the general case. By the Galois Embedding Theorem (Theorem 7.1.1 in [3]), we know that $\sqrt[\mathcal{P}^{*}]{\mathrm{H}}$ is a ring of invariants of some finite group $G \leq \mathrm{GL}(V)$ acting linearly on $\mathbb{F}[V]$. Thus

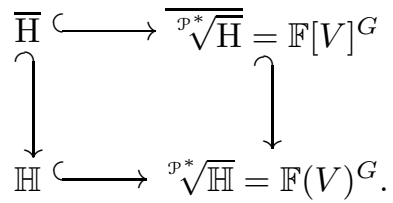

It turns out that there exists a vector space decomposition as above,

$$
V=W_{0} \oplus W_{1} \oplus \cdots \oplus W_{e},
$$

such that $G$ acts on the flags

$$
W_{0} \oplus W_{1} \oplus \cdots \oplus W_{i}
$$

for all $i=0, \ldots, e$. Moreover

$$
\begin{aligned}
& \overline{\mathrm{H}}=\left(\mathbb{F}\left[W_{0}\right] \otimes \mathbb{F}\left[W_{1}\right]^{p} \otimes \cdots \otimes \mathbb{F}\left[W_{e}\right]^{p^{e}}\right)^{G} \text { and } \\
& \mathbb{H}=\left(\mathbb{F}\left(W_{0}\right) \otimes \mathbb{F}\left(W_{1}\right)^{p} \otimes \cdots \otimes \mathbb{F}\left(W_{e}\right)^{p^{e}}\right)^{G} ;
\end{aligned}
$$

see Theorem 5.2. This extends results in 8 , Theorem II, and [3, Theorem 7.2.2, in the sense that we are able to determine the vector space dimensions of the $W_{i}$ 's and, more importantly, are able to prove that the group $G$ in question remains unchanged. In particular this means that the group $G$ must consist of flag matrices

$$
\left[\begin{array}{ccccc}
A_{0} & 0 & & \ldots & 0 \\
* & A_{1} & 0 & \ldots & 0 \\
& * & \ddots & & \vdots \\
\ldots & & \ddots & & 0 \\
* & & \ldots & * & A_{e}
\end{array}\right],
$$

where $A_{i}$ is an $m_{i} \times m_{i}$-matrix with $m_{i}=\operatorname{dim}\left(W_{i}\right)$. On the other hand, if $V$ has no basis such that $G$ consists of flag matrices, then the only purely inseparable extensions of exponent $e$ are

$$
\left(\mathbb{F}[V]^{p^{e}}\right)^{G} \subseteq \mathbb{F}[V]^{G} .
$$

In Section 6 we take a break from these constructive methods and look at homological properties of $\mathrm{H}$ and $\sqrt[\mathcal{P}^{*}]{\mathrm{H}}$. We show that $\mathrm{H}$ is Cohen-Macaulay if and only if $\sqrt[\mathcal{P}^{*}]{\mathrm{H}}$ is Cohen-Macaulay for any reduced Noetherian unstable algebra $\mathrm{H}$.

This motivates Section 7 where we look at polynomial rings. It turns out that $\sqrt[\mathcal{P}^{*}]{\mathrm{H}}$ is a polynomial algebra if and only if $\mathrm{H}$ is polynomial. Moreover, $\sqrt[\mathcal{P}^{*}]{\mathrm{H}}=$ $\mathbb{F}\left[h_{1}, \ldots, h_{n}\right]$ if and only if

$$
\mathrm{H}=\mathbb{F}\left[h_{1}, \ldots, h_{n_{0}}, h_{n_{0}+1}^{0}, \ldots, h_{n_{1}}^{p}, h_{n_{1}+1}^{p^{2}}, \ldots, h_{n_{e}}^{p^{e}}\right],
$$

where $n_{e}=n$ and $n_{i}=\operatorname{dim}_{\mathbb{F}}\left(W_{0} \oplus \cdots \oplus W_{i}\right)$. Recall that an unstable $\mathcal{P}^{*}$-inseparably closed polynomial algebra over the Steenrod algebra is the ring of invariants $\mathbb{F}[V]^{G}$ for some $G \leq \operatorname{GL}(n, \mathbb{F})$ by the Galois Embedding Theorem (Theorem 7.1.1 in [3]). Combined with the results from Section 5, this means that if $G$ consists of flag matrices, then $\mathrm{H}$ is polynomial if and only if $\sqrt[\mathcal{P}^{*}]{\mathrm{H}}$ is polynomial, and the generators are just $p$ th powers/roots of one another. However, if $G$ does not consist of flag 
matrices, then there exists no unstable algebra $\mathrm{H} \hookrightarrow \mathbb{F}[V]^{G}$ such that $\sqrt[\mathcal{P}^{*}]{\mathrm{H}}=\mathbb{F}[V]^{G}$. This solves a twenty-year-old conjecture due to Clarence Wilkerson; see Conjecture 5.1 in 8 .

Part of this work was done during my stay at the National Australian University, Canberra, in June 2003. I thank David Benson, Henning Krause, and Amnon Neeman for the invitation and their hospitality. I also thank the referee for helpful comments.

\section{ReCOlLECtions AND PRELIMINARIES}

Let $\mathrm{H}$ be an unstable reduced algebra over the Steenrod algebra of reduced powers $\mathcal{P}^{*}$. We denote the characteristic by $p$, and the order of the ground field $\mathbb{F}$ by $q$. Recall that the Steenrod algebra contains an infinite sequence of derivations iteratively defined as

$$
\begin{aligned}
& \mathscr{P}^{\Delta_{1}}=\mathscr{P}^{1}, \\
& \mathscr{P}^{\Delta_{i}}=\mathscr{P}^{\Delta_{i-1}} \mathscr{P}^{q^{i-1}}-\mathscr{P}^{q^{i-1}} \mathscr{P}^{\Delta_{i-1}} \quad \text { for } i \geq 2 .
\end{aligned}
$$

We set

$$
\mathscr{P}^{\Delta_{0}}(h)=\operatorname{deg}(h) h \quad \forall h \in \mathrm{H} .
$$

Note that $\mathscr{P} \Delta_{0}$ is not an element of the Steenrod algebra.

The algebra $\mathrm{H}$ is called $\mathcal{P}^{*}$-inseparably closed, if whenever $h \in \mathrm{H}$ and

$$
\mathscr{P}^{\Delta_{i}}(h)=0 \quad \forall i \geq 0
$$

then there exists an element $h^{\prime} \in \mathrm{H}$ such that

$$
\left(h^{\prime}\right)^{p}=h .
$$

The $\mathcal{P}^{*}$-inseparable closure of $\mathrm{H}$ is a $\mathcal{P}^{*}$-inseparably closed algebra $\sqrt[\mathcal{P}^{*}]{\mathrm{H}}$ containing $H$ such that the following universal property holds: Whenever we have a $\mathcal{P}^{*}$ inseparably closed algebra $\mathrm{H}^{\prime}$ containing $\mathrm{H}$ there exists an embedding $\varphi: \sqrt[\mathcal{P}^{*}]{\mathrm{H}} \hookrightarrow$ $\mathrm{H}^{\prime}$.

The following method to construct the $\mathcal{P}^{*}$-inseparable closure of $\mathrm{H}$ is taken from Section 4.1 in 3 . Denote by $\mathcal{C} \subseteq \mathrm{H}$ the subalgebra consisting of the $\mathscr{P}^{\Delta_{i}}$-constants for all $i \geq 0$, i.e.,

$$
\mathcal{C}=\mathcal{C}(\mathrm{H})=\left\{h \in \mathrm{H} \mid \mathscr{P}^{\Delta_{i}}(h)=0 \forall i \geq 0\right\} .
$$

It turns out that the subalgebra of constants $\mathcal{C}$ is an unstable algebra over the Steenrod algebra (Lemma 4.1.2 loc.cit.). Moreover, it is Noetherian whenever $\mathrm{H}$ is (Lemma 4.1.1 loc.cit.). By construction we have integral extensions

$$
\mathrm{H}^{p} \hookrightarrow \mathcal{C} \hookrightarrow \mathrm{H}
$$

where $\mathrm{H}^{p}=\left\{h^{p} \mid h \in \mathrm{H}\right\}$. Denote by $\mathscr{S}$ a set of generators for $\mathcal{C}$ as a module over $\mathrm{H}^{p}$. Define an algebra

$$
\mathrm{H}_{1}=\left(\mathrm{H} \otimes_{\mathbb{F}} \mathbb{F}\left[\gamma_{s} \mid s \in \mathscr{S}\right]\right) / \mathcal{R} a d\left(\gamma_{s}^{p}-s \mid s \in \mathscr{S}\right),
$$

where $\mathcal{R} a d(-)$ denotes the radical of the ideal $(-)$. Note that this construction comes with a canonical inclusion

$$
\varphi_{0}: \mathrm{H} \hookrightarrow \mathrm{H}_{1},
$$


by part (5) of Lemma 4.1.3 in [3. Since the new algebra $\mathrm{H}_{1}$ is again an unstable reduced algebra over the Steenrod algebra (see Lemma 4.1.4 loc.cit.), we can iterate the construction and obtain a nested sequence

$$
\mathrm{H}=\mathrm{H}_{0} \hookrightarrow \mathrm{H}_{1} \hookrightarrow \cdots \hookrightarrow \mathrm{H}_{i} \hookrightarrow \cdots \hookrightarrow
$$

of unstable reduced algebras over the Steenrod algebra. The colimit of this sequence is the $\mathcal{P}^{*}$-inseparable closure of $\mathrm{H}$ (Proposition 4.1 .5 loc.cit.). We recall the basic properties of $\sqrt[\mathcal{P} *]{\mathrm{H}}$ and the intermediate algebras $\mathrm{H}_{i}$.

Proposition 2.1. Consider the chain of unstable reduced algebras

$$
\mathrm{H}=\mathrm{H}_{0} \hookrightarrow \mathrm{H}_{1} \hookrightarrow \cdots \hookrightarrow \mathrm{H}_{i} \hookrightarrow \cdots \hookrightarrow \sqrt[\mathcal{P}^{*}]{\mathrm{H}} .
$$

Then the following statements hold.

(1) If one of the algebras in this chain is an integral domain, then so are the others.

(2) $\mathrm{H} \hookrightarrow \sqrt[\mathcal{P}^{*}]{\mathrm{H}}$ is an integral extension, and both algebras have the same Krull dimension.

(3) If $\mathrm{H}$ is integrally closed, then so is $\sqrt[\mathcal{P}^{*}]{\mathrm{H}}$.

(4) The following statements are equivalent.

- H is Noetherian.

- $\mathrm{H}_{i}$ is Noetherian.

- $\sqrt[P^{*}]{\mathrm{H}}$ is Noetherian.

- There exists an $r$ such that

$$
\mathrm{H}_{r}=\mathrm{H}_{r+1}=\cdots=\sqrt[\mathcal{P}^{*}]{\mathrm{H}} .
$$

Proof. For (1)-(3) see Proposition 4.2.1 in [3]. For (4) see part (2) of Lemma 4.1.3, Lemma 4.2.2, Proposition 4.2.4, and Theorem 6.3.1 loc.cit.

Lemma 2.2. Let $\mathrm{H}$ be an integral domain. If $\mathrm{H}$ is integrally closed, then the algebras $\mathrm{H}_{i}$ are also integrally closed for all $i$.

Proof. It is shown in part (5) of Proposition 4.2 .1 in $[3$ that $\sqrt[\mathcal{P} *]{\mathrm{H}}$ is integrally closed whenever $\mathrm{H}$ is integrally closed. The same argument presented there can be used to show that also the algebras $\mathrm{H}_{i}$ are also integrally closed.

In the same way the $\mathcal{P}^{*}$-inseparable closure of a field $\mathbb{K}$ over the Steenrod algebra can be constructed. So we obtain a chain of fields over the Steenrod algebra

$$
\mathbb{K}=\mathbb{K}_{0} \hookrightarrow \mathbb{K}_{1} \hookrightarrow \cdots \hookrightarrow \mathbb{K}_{i} \hookrightarrow \cdots \hookrightarrow \sqrt[\mathcal{P}^{*}]{\mathbb{K}}
$$

by adjoining successively $p$ th roots. Again the $p$ th powers are detected by the vanishing of the derivations $\mathscr{P}^{\Delta_{i}}$; see Section 2.3 in [3].

Let $\mathrm{H}$ be an unstable integral domain over the Steenrod algebra. Denote by $\mathbb{H}$ its field of fractions. We have seen in Proposition 4.2.6 in [3] that

$$
F F(\sqrt[\mathcal{P} *]{\mathrm{H}})=\sqrt[\mathcal{P} *]{\mathbb{H}}
$$

where $F F(-)$ denotes the field of fraction functor.

Our first goal is to refine this statement. For this we need the following result.

Proposition 2.3. Let $\mathrm{H}$ be an unstable integral domain. Then

$$
\mathcal{C}(\mathbb{H})=F F(\mathcal{C}(\mathrm{H})) .
$$


Proof. Let $\frac{f_{1}}{f_{2}} \in \mathcal{C}(\mathbb{H}), f_{1}, f_{2} \in \mathrm{H}$. Then there exists an element $\frac{h_{1}}{h_{2}} \in \sqrt[\mathcal{P} *]{\mathbb{H}}=$ $F F(\sqrt[\mathcal{P} *]{\mathrm{H}}), h_{1} h_{2} \in \sqrt[\mathcal{P} *]{\mathrm{H}}$, such that

$$
\frac{h_{1}^{p^{k}}}{h_{2}^{p^{k}}}=\frac{f_{1}}{f_{2}}
$$

for some $k \in \mathbb{N}_{0}$. Furthermore, since $h_{1}, h_{2} \in \sqrt[\mathcal{P}^{*}]{\mathrm{H}}$ we can choose $k$ such that $h_{1}^{p^{k}}, h_{2}^{p^{k}} \in \mathrm{H}$. By construction, $h_{1}^{p^{k}}, h_{2}^{p^{k}}$ are in the subalgebra of constants, $\mathcal{C}(\mathrm{H})$. Thus

$$
\frac{f_{1}}{f_{2}}=\frac{h_{1}^{p^{k}}}{h_{2}^{p^{k}}} \in F F(\mathcal{C}(\mathrm{H}))
$$

which shows that

$$
\mathcal{C}(\mathbb{H}) \subseteq F F(\mathcal{C}(\mathrm{H}))
$$

Conversely, let $\frac{f_{1}}{f_{2}} \in F F(\mathcal{C}(\mathrm{H}))$ with $f_{1}, f_{2} \in \mathcal{C}(\mathrm{H})$. Then

$$
\frac{f_{1}}{f_{2}} \in \mathbb{H}
$$

is a constant because

$$
\mathscr{P}^{\Delta_{i}}\left(\frac{f_{1}}{f_{2}}\right)=\frac{\mathscr{P}^{\Delta_{i}}\left(f_{1}\right) f_{2}-f_{1} \mathscr{P}^{\Delta_{i}}\left(f_{2}\right)}{f_{2}^{2}}=0
$$

for all $i \in \mathbb{N}_{0}$.

Proposition 2.4. Let $\mathrm{H}$ be an unstable integral domain over the Steenrod algebra. Denote by $\mathbb{H}$ its field of fractions. Then for all $i \in \mathbb{N}_{0}$ we have

$$
F F\left(\mathrm{H}_{i}\right)=\mathbb{H}_{i} .
$$

Proof. By induction it is enough to show the statement for $i=1$. If $\frac{h_{1}}{h_{2}} \in F F(\mathrm{H})_{1}$ for $h_{1}, h_{2} \in \mathrm{H}_{1}$, then $\frac{h_{1}^{p}}{h_{2}^{p}} \in F F\left(\mathrm{H}_{0}\right)=\mathbb{H}_{0}=\mathbb{H}$. Thus $\frac{h_{1}}{h_{2}} \in \mathbb{H}_{1}$, since $\mathbb{H}_{1}$ is obtained from $\mathbb{H}_{0}$ by adjoining all $p$ th roots. Thus $F F\left(\mathrm{H}_{1}\right) \subseteq \mathbb{H}_{1}$.

We prove the reverse inclusion. Let $h \in \mathbb{H}_{1}$. Then $h^{p} \in \mathbb{H}=F F(\mathrm{H})$. Thus by Proposition 2.3

$$
h^{p} \in \mathcal{C}(\mathbb{H})=F F(\mathcal{C}(\mathrm{H})) .
$$

Thus there exist elements $h_{1}, h_{2} \in \mathcal{C}(\mathrm{H})$ such that

$$
h^{p}=\frac{h_{1}}{h_{2}} .
$$

Moreover, since the elements $h_{1}, h_{2}$ are constants they have $p$ th roots, say $f_{1}, f_{2}$, in $\mathrm{H}_{1}$. Thus

$$
h=\frac{f_{1}}{f_{2}} \in F F\left(\mathrm{H}_{1}\right)
$$

and we are done. 
Hence we obtain chains

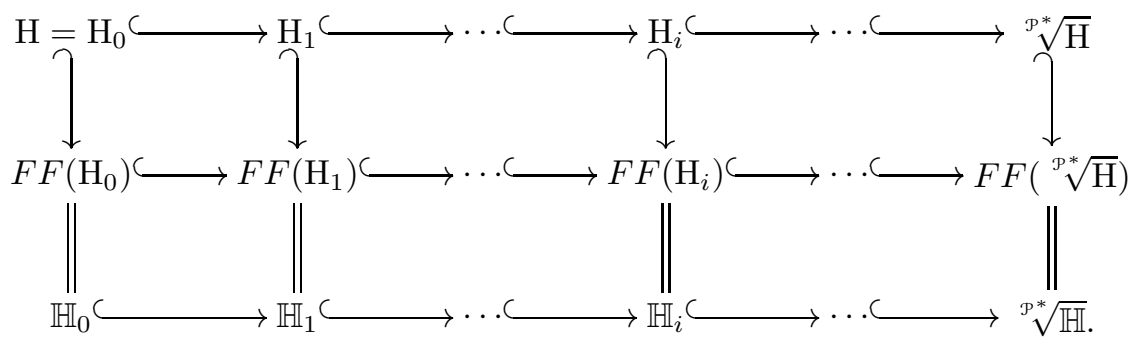

Let $\mathrm{H}$ be an unstable Noetherian integral domain over the Steenrod algebra. Then there exists an $r \in \mathbb{N}_{0}$ such that $\mathrm{H}_{r}=\sqrt[\mathcal{P} *]{\mathrm{H}}$; see Theorem 6.1.3 and Proposition 4.2.4 in [3. Also, there exists an $s \in \mathbb{N}_{0}$ such that $\mathbb{H}_{s}=\sqrt[\mathcal{P}^{*}]{\mathbb{H}}$, loc.cit. Without loss of generality we assume that $r$ and $s$ are minimal with respect to this property. Then by Proposition 4.2.4 in [3] we have that $r \geq s$. Thus for Noetherian unstable algebras we obtain finite chains

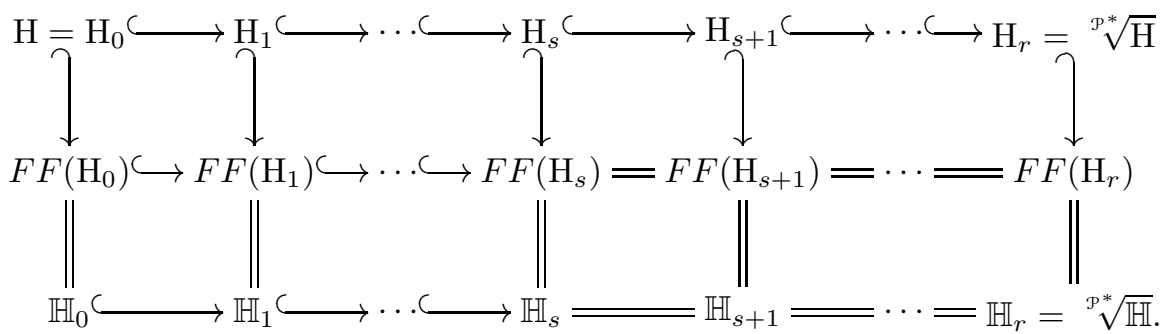

Corollary 2.5. Let $\mathrm{H}$ be an unstable Noetherian integral domain. Let $\mathrm{H}$ be integrally closed. Then with the preceding notation, $r=s$.

Proof. By Proposition 2.4, $F F\left(\mathrm{H}_{i}\right)=\mathbb{H}_{i}$. Moreover, $\mathrm{H}_{i}$ is integrally closed for all $i$ by Lemma 2.2. Thus for all $i$ the unstable part of $F F\left(\mathrm{H}_{i}\right)$ is

$$
\mathcal{U}\left(F F\left(\mathrm{H}_{i}\right)\right)=\mathrm{H}_{i}
$$

see Theorem 2.4 in [4. Thus

$$
\mathrm{H}_{s}=\mathcal{U} n\left(F F\left(\mathrm{H}_{s}\right)\right)=\mathcal{U} n\left(F F\left(\mathrm{H}_{r}\right)\right)=\mathrm{H}_{r}
$$

as desired.

\section{INSEPARABLE EXTENSIONS OF EXPONENT 1}

Let $\mathrm{H}$ be an unstable Noetherian reduced algebra over the Steenrod algebra. Define the H-module

$$
\operatorname{Der}_{\mathrm{H}}=\operatorname{span}_{\mathrm{H}}\left\{\mathscr{P}^{\Delta_{i}} \mid i \in \mathbb{N}_{0}\right\} .
$$

Then Der $_{\mathrm{H}}$ is free as a module over H; see Proposition 1.1.7 and Theorem 1.2.1 in [3. 3 Moreover it is a restricted Lie algebra of derivations acting on $\mathrm{H}$; cf. Section 2.4 in [3]. We denote by

$$
\mathcal{C}_{\text {Der }_{\mathrm{H}}}(\mathrm{H})=\left\{h \in \mathrm{H} \mid \mathscr{P}^{\Delta_{i}}(h)=0 \forall i\right\} \subseteq \mathrm{H}
$$

the subalgebra of constants with respect to the derivations in $\operatorname{Der}_{H}$.

\footnotetext{
${ }^{3}$ The module $\operatorname{Der}_{H}$ is the module $\Delta(\mathrm{H})$ in this reference.
} 
Remark. In Section 2 we called the subalgebra of constants just $\mathcal{C}=\mathcal{C}(\mathrm{H})$. For what follows however, we need to keep track of the module of derivations that is used.

Clearly,

$$
\mathrm{H}^{p} \subseteq \mathcal{C}_{\text {Der }_{\mathrm{H}}}
$$

as $\mathscr{P}^{\Delta_{i}}\left(h^{p}\right)=0$ for all $h \in \mathrm{H}$ and $i \in \mathbb{N}_{0}$. Since the extension

$$
\mathrm{H}^{p} \subseteq \mathrm{H}
$$

is purely inseparable of exponent one, so is the extension

$$
\mathrm{H}^{p} \subseteq \mathcal{C}_{\text {Der }_{\mathrm{H}}}(\mathrm{H}) .
$$

Thus

$$
\sqrt[\mathcal{P} *]{\mathrm{H}^{p}}=\sqrt[\mathcal{P}^{*}]{\mathcal{C}_{\text {DerH }_{\mathrm{H}}(\mathrm{H})}}=\sqrt[\mathcal{P}^{*}]{\mathrm{H}}
$$

Proposition 3.1. Let $\mathrm{H}$ be an unstable reduced algebra over the Steenrod algebra. Then $\mathrm{H}$ is $\mathcal{P}^{*}$-inseparably closed if and only if

$$
\mathcal{C}_{\text {DerH }_{\mathrm{H}}}(\mathrm{H})=\mathrm{H}^{p} \text {. }
$$

Proof. Assume that $\mathrm{H}$ is $\mathcal{P}^{*}$-inseparably closed. By what we have done so far we know that $\mathrm{H}^{p} \subseteq \mathcal{C}_{\text {Der }_{\mathrm{H}}}(\mathrm{H})$. To prove the reverse inclusion, let $h \in \mathcal{C}_{\text {Der }_{\mathrm{H}}}(\mathrm{H})$. Then $\mathscr{P}^{\Delta_{i}}(h)=0$ for all $i \in \mathbb{N}_{0}$. Thus there exists an element

$$
f \in \sqrt[\mathcal{P} *]{\mathcal{C}_{\text {DerH }_{\mathrm{H}}(\mathrm{H})}}=\sqrt[\mathcal{P} *]{\mathrm{H}}=\mathrm{H}
$$

such that $f^{p^{k}}=h$. Since $f^{p^{k}} \in \mathrm{H}^{p}$ for all $f \in \mathrm{H}$, we have $h=f^{p^{k}} \in \mathrm{H}^{p}$, and hence

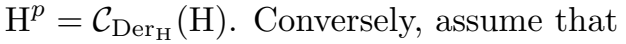

$$
\mathrm{H}^{p}=\mathcal{C}_{\text {Der }_{\mathrm{H}}}(\mathrm{H}) \subseteq \mathrm{H} \subseteq \sqrt[\mathcal{P}^{*}]{\mathrm{H}} .
$$

Let $h \in \sqrt[\mathcal{P}^{*}]{\mathrm{H}}$. Thus there exists a $k \in \mathbb{N}_{0}$ such that $h^{p^{k}} \in \mathrm{H}$. Since $\operatorname{Der}_{\mathrm{H}}$ vanishes on $p$ th powers we find that

$$
h^{p^{k}} \in \mathcal{C}_{\operatorname{Der}_{\mathrm{H}}}(\mathrm{H})=\mathrm{H}^{p} .
$$

Thus $h^{p^{k-1}} \in \mathrm{H}$. Iteratively we find that $h \in \mathrm{H}$, i.e., $\mathrm{H}$ is $\mathcal{P}^{*}$-inseparably closed.

Corollary 3.2. We have

$$
\mathcal{C}_{\operatorname{Der}_{\mathbb{F}[V]}}(\mathbb{F}[V])=\mathbb{F}[V]^{p}=\mathbb{F}\left[x_{1}^{p}, \ldots, x_{n}^{p}\right] .
$$

Proof. Since $\mathbb{F}[V]$ is $\mathcal{P}^{*}$-inseparably closed (see Corollary 4.2 .8 in 3$]$ ), this result is an immediate corollary of Proposition 3.1.

We recall some facts about $\operatorname{Der}_{\mathrm{H}}$ and its action on $\mathrm{H}$. First the Lie algebra structure is particularly simple, namely

$$
\left[\mathscr{P}^{\Delta_{i}}, \mathscr{P}^{\Delta_{j}}\right]= \begin{cases}0 & \text { if } i, j>0, \\ \mathscr{P}^{\Delta_{i}} & \text { if } i \neq 0 \text { and } j=0, \\ -\mathscr{P}^{\Delta_{j}} & \text { if } i=0 \text { and } j \neq 0\end{cases}
$$

(see the remark on page 12 of [3]), and

$$
\left(\mathscr{P}^{\Delta_{i}}\right)^{p}=0
$$


(see Section 2.4 in $\left[3\right.$ ). The $\Delta$-length of $\mathrm{H}$ is defined to be the smallest integer $\lambda_{\mathrm{H}}$ such that the derivation

$$
h_{0} \mathscr{P}^{\Delta_{i_{0}}}+\cdots+h_{\lambda} \mathscr{P}^{\Delta_{i_{\lambda}}}
$$

vanishes on $\mathrm{H}$ for some $h_{0}, \ldots, h_{\lambda} \in \mathrm{H}$ and $i_{0}, \ldots, i_{\lambda} \in \mathbb{N}_{0}$ (see Section 1.2 in [3]) 5 In this case, any $\lambda+1$ derivations are linearly dependent over $\mathrm{H}$ (Proposition 1.1.7 in [3]). The $\Delta$-length $\lambda_{\mathrm{H}}$ is at most the Krull dimension of $\mathrm{H}$ over $\mathbb{F}$ (cf. Corollary 1.2.2 in [3]). Moreover, the coefficients can be chosen to be

$$
h_{i}=(-1)^{i} \mathbf{d}_{\lambda, i}
$$

(up to a sign) the Dickson classes in dimension $\lambda$ (see Theorems 5.1.9 and 5.2.1 in [3]). Note that by convention $\mathbf{d}_{\lambda, \lambda}=1$. Then the normalized equation

$$
\left(\mathbf{d}_{\lambda, 0} \mathscr{P}^{\Delta_{0}}-\cdots+(-1)^{\lambda} \mathbf{d}_{\lambda, \lambda} \mathscr{P}^{\Delta_{\lambda}}\right)(h)=0 \quad \forall h \in \mathrm{H}
$$

is called the $\Delta$-relation of $\mathrm{H}$. By abuse of notation, we also call the element

$$
\mathbf{d}_{\mathrm{H}}=\mathbf{d}_{\lambda, 0} \mathscr{P}^{\Delta_{0}}-\cdots+(-1)^{\lambda} \mathbf{d}_{\lambda, \lambda} \mathscr{P}^{\Delta_{\lambda}} \in \operatorname{Der}_{\mathrm{H}}
$$

the $\Delta$-relation for $\mathrm{H} 6$ Finally we note that the $\Delta$-length of $\mathrm{H}$ is equal to its Krull dimension if $\mathrm{H}$ is $\mathcal{P}^{*}$-inseparably closed (cf. Theorem 8.1.5 in [3]). The converse is not quite true as the following example taken from Section 7.4 in [3] shows.

Example 3.3. Consider the field $\mathbb{F}=\mathbb{F}_{2}$ with two elements, and take a polynomial algebra in two linear generators $x, y, \mathbb{F}[x, y]$. The Dickson algebra in this case is

$$
\mathcal{D}(2)=\mathbb{F}\left[x^{2} y+x y^{2}, x^{2}+y^{2}+x y\right] \hookrightarrow \mathbb{F}[x, y] .
$$

Define an intermediate algebra $\mathrm{H}$ by

$$
\mathrm{H}=\mathbb{F}\left[x^{2}+y^{2}, x y, x y(x+y)\right] /\left(\left(x^{2}+y^{2}\right)(x y)+(x y(x+y))^{2}\right) .
$$

Then $\mathrm{H}$ is an unstable integral domain, but it is not $\mathcal{P}^{*}$-inseparably closed because

$$
\mathscr{P}^{\Delta_{i}}\left(x^{2}+y^{2}\right)=0 \quad \forall i \geq 0, \text { but } x+y \notin \mathrm{H} .
$$

However, its $\Delta$-relation

$$
\mathbf{d}_{\mathrm{H}}=\mathbf{d}_{2,0} \mathscr{P}^{\Delta_{0}}-\mathbf{d}_{2,1} \mathscr{P}^{\Delta_{1}}+\mathscr{P}^{\Delta_{2}}
$$

has length 2, which is equal to its Krull dimension. Note that the field of fractions of $\mathrm{H}$,

$$
F F(\mathrm{H})=\mathbb{F}(x+y, x y),
$$

is inseparably closed. Therefore the algebra $\mathrm{H}$ is not integrally closed because $x+y \notin \mathrm{H}$ (cf. Corollary 2.5).

Proposition 3.4. Let $\mathrm{H}$ be an unstable Noetherian integral domain. If the $\Delta$-length $\lambda_{\mathrm{H}}$ is equal to the Krull dimension $n$ of $\mathrm{H}$, then

$$
\sqrt[\mathcal{P} *]{\mathrm{H}} \subseteq \overline{\mathrm{H}}
$$

where $\overline{(-)}$ denotes the integral closure.

\footnotetext{
${ }^{4}$ If there is no possible confusion we will omit the subscript and just write $\lambda=\lambda_{\mathrm{H}}$.

${ }^{5}$ If $\lambda_{\mathrm{H}} \in \mathbb{N}_{0}$ exists, then $\mathrm{H}$ is called $\Delta$-finite. This is a weaker condition than Noetherianess. For example the polynomial algebra $\mathbb{F}\left[x_{1}^{p}, x_{2}^{p}, \ldots\right]$ in infinitely many generators has $\Delta$-length zero, but it is not Noetherian.

${ }^{6} \mathrm{We}$ will suppress the subscript, and write $\mathbf{d}$ for $\mathbf{d}_{\mathrm{H}}$ if no confusion is possible.
} 
Proof. Since the $\Delta$-length of $\mathrm{H}$ is equal to its Krull dimension, we have integral extensions

$$
\mathcal{D}(n) \hookrightarrow \mathrm{H} \hookrightarrow \sqrt[\mathcal{P} *]{\mathrm{H}} \hookrightarrow \mathbb{F}[V]
$$

by the Little Imbedding Theorem (Theorem 7.4.4 in [3]) and the Embedding Theorem (Corollary 6.1.5 loc.cit.). The corresponding extensions of the field of fractions are Galois extensions, so in particular separable. Since $F F(\mathrm{H}) \subseteq F F(\sqrt[\mathcal{P *}]{\mathrm{H}})$ is also purely inseparable, we have $F F\left(\sqrt[\mathcal{P}^{*}]{\mathrm{H}}\right)=F F(\mathrm{H})=F F(\overline{\mathrm{H}})$. Thus $F F(\mathrm{H})=\mathbb{F}(V)^{G}$ for some group $G \leq \mathrm{GL}(V)$. Therefore, $\overline{\mathrm{H}}=\mathbb{F}[V]^{G}$ is inseparably closed. Hence $\sqrt[\mathcal{P}]{\mathrm{H}} \hookrightarrow \overline{\mathrm{H}}$ by the universal property of the inseparable closure.

Remark. Note that it follows from the preceding result that if $\mathrm{H}$ is integrally closed and the $\Delta$-length is equal to its Krull dimension, then

$$
\sqrt[\mathcal{P}^{*}]{\mathrm{H}}=\overline{\mathrm{H}}=\mathrm{H}
$$

We want to investigate purely inseparable extensions $\mathrm{H} \hookrightarrow \mathbb{F}[V]$ of exponent one, i.e., we have

$$
\mathbb{F}[V]^{p} \hookrightarrow \mathrm{H} \hookrightarrow \mathbb{F}[V] .
$$

For this we turn our attention to the corresponding extensions of fields of fractions

$$
\mathbb{F}(V)^{p} \hookrightarrow \mathbb{H} \hookrightarrow \mathbb{F}(V) .
$$

Let $\operatorname{Der}_{\mathbb{H}}$ be the vector space over $\mathbb{H}$ generated by the elements $\mathscr{P}^{\Delta_{i}}$ for $i \in \mathbb{N}_{0}$. Since the relations and are intrinsic of the Steenrod algebra, the vector space $\operatorname{Der}_{\mathbb{H}}$ is also a restricted Lie algebra. Thus any vector subspace of $\operatorname{Der}_{\mathbb{H}}$ is a restricted Lie subalgebra and vice versa.

Proposition 3.5. Let $\mathrm{H}$ be an unstable integral domain and $\mathbb{H}$ its field of fractions. The vector space $\mathrm{Der}_{\mathbb{H}}$ satisfies the following properties.

(1) The elements $\mathscr{P}^{\Delta_{i}}$ are derivations on $\mathbb{H}$.

(2) The $\Delta$-relation $\mathbb{H}$ is well defined, and coincides with the $\Delta$-relation on $\mathrm{H}$. In particular, the $\Delta$-lengths are equal.

Proof. $\mathrm{AD}(1)$ : The action of $\mathscr{P}^{\Delta_{i}}$ on $\mathbb{H}$ is given by the formula

$$
\mathscr{P}^{\Delta_{i}}\left(\frac{f_{1}}{f_{2}}\right)=\frac{\mathscr{P}^{\Delta_{i}}\left(f_{1}\right) f_{2}-f_{1} \mathscr{P}^{\Delta_{i}}\left(f_{2}\right)}{f_{2}^{2}}
$$

for any $f_{1}, f_{2} \in \mathrm{H}$. Thus they are derivations on the field of fractions also.

$\operatorname{AD}(2)$ : The set $\operatorname{Der}_{\mathbb{H}}$ is a vector space by construction. Let $\lambda_{\mathbb{H}}$ be its dimension. Then any $\lambda_{\mathbb{H}}+1$ elements are linearly independent. Thus the $\Delta$-length is $\lambda_{\mathbb{H}}$ with $\Delta$-relation

$$
d=f_{0} \mathscr{P}^{\Delta_{0}}+\cdots+f_{\lambda} \mathscr{P}^{\Delta_{\lambda}} .
$$

Without loss of generality we can assume that the coefficients $f_{i} \in \mathrm{H}$ for all $i$. Thus $\lambda_{\mathbb{H}}$ is at least equal to the $\Delta$-length, $\lambda_{\mathrm{H}}$, of $\mathrm{H}$. On the other hand, if $\mathbf{d}_{\mathrm{H}}$ is a $\Delta$-relation for $\mathrm{H}$, then by

$$
\mathbf{d}_{\mathrm{H}}\left(\frac{f_{1}}{f_{2}}\right)=\frac{\mathbf{d}_{\mathrm{H}}\left(f_{1}\right) f_{2}-f_{1} \mathbf{d}_{\mathrm{H}}\left(f_{2}\right)}{f_{2}^{2}}=0
$$

$\mathbf{d}_{\mathrm{H}}$ vanishes also on $\mathbb{H}$. Thus $\lambda_{\mathbb{H}} \leq \lambda_{\mathrm{H}}$. Therefore $\lambda_{\mathbb{H}}=\lambda_{\mathrm{H}}$ and $\mathbf{d}_{\mathbb{H}}=\mathbf{d}_{\mathrm{H}}$.

Corollary 3.6. Let $\mathrm{H}$ be an unstable integral domain and $\overline{\mathrm{H}}$ its integral closure. Then

$$
\lambda_{\mathrm{H}}=\lambda_{\overline{\mathrm{H}}} \quad \text { and } \quad \mathbf{d}_{\mathrm{H}}=\mathbf{d}_{\overline{\mathrm{H}}} .
$$


Proof. By Proposition 3.5. part (2), $\Delta$-lengths, as well as the $\Delta$-relations of $\mathrm{H}$ and its field of fractions, coincide. Since $\mathrm{H}$ and $\overline{\mathrm{H}}$ have the same field of fractions we are done.

Lemma 3.7. Let $\mathrm{H}^{\prime} \subseteq \mathrm{H}$ be unstable reduced Noetherian algebras over the Steenrod algebra. Denote by $\lambda_{\mathrm{H}^{\prime}}$, resp. $\lambda_{\mathrm{H}}$, the $\Delta$-length of $\mathrm{H}^{\prime}$, resp. $\mathrm{H}$. Then $\lambda_{\mathrm{H}^{\prime}} \leq \lambda_{\mathrm{H}}$.

Proof. By Corollary 3.6 the $\Delta$-length and $\Delta$-relation of an unstable algebra $\mathrm{H}$ and its integral closure $\overline{\mathrm{H}}$ are equal. Thus without loss of generality we assume that $\mathrm{H}^{\prime}$ and $\mathrm{H}$ are integrally closed.

Denote by $\mathcal{D}(l)$ the Dickson algebra of dimension $l$. By Theorem 5.1.9 in 3

$$
\mathcal{D}\left(\lambda_{\mathrm{H}}\right) \hookrightarrow \mathbb{H}
$$

is a maximal Dickson algebra in $\mathbb{H}$. Applying the same theorem for $\mathrm{H}^{\prime}$ gives

$$
\mathcal{D}\left(\lambda_{\mathrm{H}^{\prime}}\right) \hookrightarrow \mathbb{H}^{\prime} \hookrightarrow \mathbb{H} .
$$

Since $\mathcal{D}\left(\lambda_{\mathrm{H}}\right)$ is the maximal Dickson algebra in $\mathbb{H}$, we find that $\lambda_{\mathbb{H}^{\prime}} \leq \lambda_{\mathbb{H}}$ as desired.

Lemma 3.8. Let $U$ and $W$ be finite dimensional vector spaces over $\mathbb{F}$. We note that the $\Delta$-length $\lambda$ of $\mathbb{F}[U] \otimes \mathbb{F}[W]^{p^{t}}, t>0$, is equal to the vector space dimension of $U$ with $\Delta$-relation

$$
\mathbf{d}=\mathbf{d}_{\lambda, 0} \mathscr{P}^{\Delta_{0}}-\cdots+(-1)^{\lambda} \mathbf{d}_{\lambda, \lambda} \mathscr{P}^{\Delta_{\lambda}},
$$

where $\mathbb{F}[U]^{\mathrm{GL}(\lambda, \mathbb{F})}=\mathbb{F}\left[\mathbf{d}_{\lambda, 0}, \ldots, \mathbf{d}_{\lambda, \lambda-1}\right]$.

Proof. The element $\mathbf{d}$ is a $\Delta$-relation for $\mathbb{F}[U]$ by Theorem 1.2 .3 in [3]. Since $\mathscr{P} \Delta_{i}$ vanishes on $p$ th powers for all $i \in \mathbb{N}_{0}$, the element $\mathbf{d}$ vanishes on $\mathbb{F}[U] \otimes \mathbb{F}[W]^{p^{t}}$. So, $\lambda \leq \operatorname{dim}_{\mathbb{F}}(U)$.

On the other hand, $\mathbb{F}[U] \hookrightarrow \mathbb{F}[U] \otimes \mathbb{F}[W]^{p^{t}}$. Therefore by Lemma 3.7 the $\Delta$-length is at least $\operatorname{dim}_{\mathbb{F}}(U)$, and we are done.

Corollary 3.9. The $\Delta$-length $\lambda$ of $\mathbb{F}(U) \otimes \mathbb{F}(W)^{p^{t}}$ is equal to the vector space dimension of $U$, for $t \geq 1$. The subfield of constants is

$$
\mathcal{C}_{\operatorname{Der}_{\mathbb{P}(U) \otimes \mathbb{F}(W)^{p}}}\left(\mathbb{F}(U) \otimes \mathbb{F}(W)^{p^{t}}\right)=\mathbb{F}(U)^{p} \otimes \mathbb{F}(W)^{p^{t}} .
$$

Moreover, the $\Delta$-relation is

$$
\mathbf{d}=\mathbf{d}_{\lambda, 0} \mathscr{P}^{\Delta_{0}}+\cdots+(-1)^{\lambda} \mathbf{d}_{\lambda, \lambda} \mathscr{P}^{\Delta_{\lambda}} .
$$

Proof. This is immediate from part (2) of Proposition 3.5. Lemma 3.8, and Corollary 3.2 .

Since the $\Delta$-relation of $\mathbb{F}(V)$ has length $n=\operatorname{dim}_{\mathbb{F}}(V)$, we have $\operatorname{dim}_{\mathbb{F}(V)}\left(\operatorname{Der}_{\mathbb{F}(V)}\right)$ $=n$ and

$$
\operatorname{Der}_{\mathbb{F}(V)}=\operatorname{span}_{\mathbb{F}(V)}\left\{\mathscr{P}^{\Delta_{0}}, \ldots, \mathscr{P}^{\Delta_{n-1}}\right\} .
$$

Moreover, the index over the subfield of constants is

$$
\left[\mathbb{F}(V): \mathbb{F}(V)^{p}\right]=p^{n} .
$$

Thus we can apply the structure theorem for purely inseparable extensions of exponent one (see, e.g., Chapter IV, Section 8 in [1]). It tells us that

$$
\mathbb{H} \subseteq \mathbb{F}(V)
$$


is a purely inseparable extension of exponent one if and only if there exists a restricted Lie subalgebra $\mathrm{D} \subseteq \operatorname{Der}_{\mathbb{F}(V)}$ such that

$$
\mathbb{H}=\mathcal{C}_{\mathrm{D}}(\mathbb{F}(V)) .
$$

So, take a subspace $\mathrm{D} \subseteq \operatorname{Der}_{\mathbb{F}(V)}$. We recall from Corollary 3.9 that

$$
\mathbb{F}(V)=\mathcal{C}_{\mathrm{D}}(\mathbb{F}(V)) \text { for } \mathrm{D}=0
$$

and

$$
\mathbb{F}(V)^{p}=\mathcal{C}_{\mathrm{D}}(\mathbb{F}(V)) \quad \text { for } \mathrm{D}=\operatorname{Der}_{\mathbb{F}(V)} .
$$

Thus we are left to characterize those $\mathrm{D} \subseteq \operatorname{Der}_{\mathbb{F}(V)}$ such that $\mathbb{H}=\mathcal{C}_{\mathrm{D}}(\mathbb{F}(V))$ carries a $\mathcal{P}^{*}$-module structure.

Proposition 3.10. Let $\mathbb{K} \hookrightarrow \mathbb{F}(V)$ be a field over the Steenrod algebra. Let $\mathbf{d}$ be the $\Delta$-relation of $\mathbb{K}$ with $\Delta$-length $\lambda$. Then the vector space of derivations vanishing on $\mathbb{K}$,

$$
\mathrm{D}_{\mathbb{K}}=\operatorname{span}_{\mathbb{F}}\left\{d \in \operatorname{Der}_{\mathbb{F}(V)}|d|_{\mathbb{K}}=0\right\},
$$

has dimension $n-\lambda$, where $n=\operatorname{dim}_{\mathbb{F}}(V)$.

Proof. The $\Delta$-relation of $\mathbb{K}$ is

$$
\mathbf{d}=\mathbf{d}_{\lambda, 0} \mathscr{P}^{\Delta_{0}}+\cdots+(-1)^{\lambda} \mathbf{d}_{\lambda, \lambda} \mathscr{P}^{\Delta_{\lambda}} .
$$

By Proposition 1.1.7 in [3] any $\lambda+1$ derivations in $\operatorname{Der}_{\mathbb{F}(V)}$ are linearly dependent. Moreover by Lemma 1.1.8 loc.cit. we find that in particular the $n-\lambda$ elements

$$
\mathbf{d}_{i}=\mathbf{d}_{\lambda, 0}^{q^{i}} \mathscr{P}^{\Delta_{i}}+\cdots+(-1)^{\lambda} \mathbf{d}_{\lambda, \lambda}^{q^{i}} \mathscr{P}^{\Delta_{\lambda+1}}
$$

for $i=0, \ldots, n-\lambda-1$ vanish on $\mathbb{K}$. Since the $\mathbf{d}_{i}$ 's are linearly independent in $\operatorname{Der}_{\mathbb{F}(V)}$ we have that

$$
\operatorname{dim}\left(\mathrm{D}_{\mathbb{K}}\right) \geq n-\lambda .
$$

On the other hand, if $d \in \mathrm{D}_{\mathbb{K}}$, then

$$
d=f_{0} \mathscr{P}^{\Delta_{0}}+\cdots+f_{n-1} \mathscr{P}^{\Delta_{n-1}} .
$$

Then there are $k_{0}, \ldots, k_{n-1-\lambda}$ such that

$$
d-\sum_{i=0}^{n-1-\lambda} k_{i} \mathbf{d}_{i}=f_{0}^{\prime} \mathscr{P}^{\Delta_{0}}+\cdots+f_{\lambda-1}^{\prime} \mathscr{P}^{\Delta_{\lambda-1}}
$$

for some $f_{0}^{\prime}, \ldots, f_{\lambda-1}^{\prime} \in \mathbb{K}$. Thus if $d$ were linearly independent of the $\mathbf{d}_{i}$ 's, then the expression (困) is not zero. This in turn means that there is a relation on $\mathbb{K}$ shorter than the $\Delta$-relation. This is a contradiction. Therefore $\operatorname{dim}\left(\mathrm{D}_{\mathbb{K}}\right)=n-\lambda$.

Theorem 3.11. The extension $\mathbb{H} \subseteq \mathbb{F}(V)$ is a purely inseparable extension of exponent one of fields over the Steenrod algebra if and only if

$$
\mathbb{H}=\mathbb{F}\left(x_{1}, \ldots, x_{k}, x_{k+1}^{p}, \ldots, x_{n}^{p}\right)=\mathbb{F}(U) \otimes \mathbb{F}(V / U)^{p}
$$

for some $k \in\{1, \ldots, n\}$ and $\operatorname{dim}(U)=k$. Furthermore, in this case

$$
\mathbb{H}=\mathcal{C}_{\mathrm{D}}(\mathbb{F}(V))
$$

where $\mathrm{D}$ has vector space dimension $n-k$. If $k<n$, then $\mathrm{D}$ is generated by the $\Delta$-relation of $\mathbb{H}$,

$$
\mathbf{d}_{\mathbb{H}}=\mathbf{d}_{k, 0} \mathscr{P}^{\Delta_{0}}+\cdots+(-1)^{k} \mathbf{d}_{k, k} \mathscr{P}^{\Delta_{k}}
$$


and its translates

$$
\mathbf{d}_{i}=\mathbf{d}_{k, 0}^{q^{i}} \mathscr{P}^{\Delta_{i}}+\cdots+(-1)^{k} \mathbf{d}_{k, k}^{q^{i}} \mathscr{P}^{\Delta_{k+i}}
$$

for $i=1, \ldots, n-k-1$.

Proof. If

$$
\mathbb{H}=\mathbb{F}\left(x_{1}, \ldots, x_{k}, x_{k+1}^{p}, \ldots, x_{n}^{p}\right),
$$

then it is clearly a field over the Steenrod algebra. Moreover,

$$
\mathbb{F}\left(x_{1}, \ldots, x_{k}, x_{k+1}^{p}, \ldots, x_{n}^{p}\right)=\mathcal{C}_{\mathrm{D}}(\mathbb{F}(V))
$$

for $\mathrm{D}$ generated by the $\Delta$-relation of $\mathbb{H}$ and its translates $\mathbf{d}_{i}$ of length $\lambda_{\mathrm{H}}=k$ (see Corollary 3.9 and Proposition 3.10 .

We prove the converse. Set $\lambda=\lambda_{\mathbb{H}}$. Let $\mathbf{d}$ be the $\Delta$-relation of $\mathbb{H}$. Then

$$
\mathbf{d}=\mathbf{d}_{\lambda, 0} \mathscr{P}^{\Delta_{0}}+\cdots+(-1)^{\lambda} \mathbf{d}_{\lambda, \lambda} \mathscr{P}^{\Delta_{\lambda}}
$$

vanishes on $\mathbb{H}$. Let $U \leq V$ be a vector subspace of dimension $\lambda$. We also note that the field $\mathbb{F}(U) \otimes \mathbb{F}(V / U)^{p}$ has $\Delta$-relation $\mathbf{d}$ and $\Delta$-length $\lambda$ by Corollary 3.9. Certainly,

$$
\mathbb{F}(V)^{p} \hookrightarrow \mathbb{F}(U) \otimes \mathbb{F}(V / U)^{p} \hookrightarrow \mathbb{F}(V)
$$

is a purely inseparable extension of exponent one. We show that $\mathbb{F}(U) \otimes \mathbb{F}(V / U)^{p} \hookrightarrow$ $\mathbb{H}$. Since $\mathbb{H} \hookrightarrow \mathbb{F}(V)$ is purely inseparable of exponent one, we have

$$
\mathbb{F}(V / U)^{p} \hookrightarrow \mathbb{H} .
$$

Since the coefficients of the $\Delta$-relation are the Dickson classes, we know that $F F(\mathcal{D}(\lambda)) \hookrightarrow \mathbb{H}$. Thus

$$
F F(\mathcal{D}(\lambda)) \otimes \mathbb{F}(V / U)^{p} \hookrightarrow \mathbb{H} .
$$

Since $\mathbb{H} \hookrightarrow \mathbb{F}(V)$ is purely inseparable, we find that the separable closure of $F F(\mathcal{D}(\lambda)) \otimes \mathbb{F}[V / U]^{p}$ is in $\mathbb{H}$. This in turn is just

$$
\mathbb{F}(U) \otimes \mathbb{F}(V / U)^{p} \hookrightarrow \mathbb{H} .
$$

Obviously $\left|\mathbb{F}(V): \mathbb{F}(U) \otimes \mathbb{F}(V / U)^{p}\right|=p^{n-\lambda}$. By Theorem 19 on page 186 of [1] we have that also

$$
|\mathbb{F}(V): \mathbb{H}|=p^{n-\lambda}
$$

because $\mathcal{D}_{\mathbb{H}}$ has dimension $n-\lambda$ (Proposition 3.10). Hence $\mathbb{H}=\mathbb{F}(U) \otimes \mathbb{F}(V / U)^{p}$ as desired.

Corollary 3.12. Let $\mathrm{H} \subseteq \mathbb{F}[V]$ be a purely inseparable extension of exponent one. Let $\mathrm{H}$ be integrally closed. Then $\mathrm{H}$ is an unstable algebra over the Steenrod algebra if and only if $\mathrm{H}=\mathbb{F}\left[x_{1}, \ldots, x_{\lambda}, x_{\lambda+1}^{p}, \ldots, x_{n}^{p}\right]$, where $\lambda=\lambda_{\mathrm{H}}$ is the $\Delta$-length of $\mathrm{H}$.

Proof. If $\mathrm{H}$ is an unstable algebra over the Steenrod algebra, then $\mathbb{H}$ is a field over the Steenrod algebra. Moreover, since $\mathrm{H} \hookrightarrow \mathbb{F}[V]$ has exponent one, so has the extension $\mathbb{H} \hookrightarrow \mathbb{F}(V)$. Thus $\mathbb{H}=\mathbb{F}(U) \otimes \mathbb{F}(V / U)^{p}$ for $\operatorname{dim}_{\mathbb{E}}(U)=\lambda$ by Theorem 3.11 Hence by Theorem 2.4 in [4]

$$
\mathrm{H}=\overline{\mathrm{H}}=\mathcal{U} n(\mathbb{H})=\mathbb{F}[U] \otimes \mathbb{F}[V / U]^{p} .
$$

Conversely, the algebra $\mathbb{F}[U] \otimes \mathbb{F}[V / U]^{p}$ is certainly an unstable algebra over the Steenrod algebra. 
Remark. For any unstable integral domain $\mathrm{H}$ its integral closure $\overline{\mathrm{H}}$ also carries an unstable $\mathcal{P}^{*}$-module structure because $\overline{\mathrm{H}}=\mathcal{U} n(\mathbb{H})$ (see Theorem 2.4 in [4]). The converse is not true as we illustrate with the next example.

Example 3.13. Let $\mathbb{F}$ be the prime field of characteristic 2 and let $A$ be the subalgebra of $\mathbb{F}[x, y]$ generated by $x, x y, y^{3}$. Then $\mathrm{A} \hookrightarrow \mathbb{F}[x, y]$ is an integral extension. Moreover, $F F(\mathrm{~A})=\mathbb{F}(x, y)$. Therefore $\overline{\mathrm{A}}=\mathbb{F}[x, y]$ is an unstable algebra over the Steenrod algebra. However A does not carry a $\mathcal{P}^{*}$-module structure because

$$
\mathscr{P}^{1}(x y)=x^{2} y+x y^{2} \notin \mathrm{A},
$$

as the only elements of degree 3 in $\mathrm{A}$ are $x^{3}, x^{2}, y^{3}$.

Thus the assumption $\mathrm{H}=\overline{\mathrm{H}}$ cannot be dropped in the preceding result.

Corollary 3.14. Let $U \leq V$. Denote $m=\operatorname{dim}_{\mathbb{F}}(U) \leq n=\operatorname{dim}_{\mathbb{F}}(V)$. Then

$$
\mathbb{F}[U] \otimes \mathbb{F}[V / U]^{p} \hookrightarrow \mathbb{F}[V]
$$

is the largest unstable subalgebra with $\Delta$-length equal to $m$.

Proof. Certainly, $\mathbb{F}[U] \otimes \mathbb{F}[V / U]^{p}$ has $\Delta$-length $m$. Let

$$
\mathbb{F}[U] \times \mathbb{F}[V / U]^{p} \hookrightarrow \mathrm{H} \hookrightarrow \mathbb{F}[V]
$$

be an intermediate unstable algebra with $\lambda_{\mathrm{H}}=m$. Since the extension (因) is purely inseparable of exponent one, we have that

$$
\mathbb{H}=\mathbb{F}\left(U^{\prime}\right) \otimes \mathbb{F}\left(V / U^{\prime}\right)^{p}
$$

for some $U^{\prime} \geq U$. But

$$
\operatorname{dim}_{\mathbb{F}}(U)=m=\lambda_{\mathbb{H}}=\operatorname{dim}_{\mathbb{F}}\left(U^{\prime}\right)
$$

and therefore $U=U^{\prime}$. Hence

$$
\mathbb{F}[U] \otimes \mathbb{F}[V / U]^{p} \subseteq \mathrm{H} \subseteq \mathcal{U} n(\mathrm{H})=\overline{\mathrm{H}}=\mathbb{F}[U] \otimes \mathbb{F}[V / U]^{p}
$$

gives the desired result.

\section{Purely inseparable extensions of arbitrary exponent}

In this section we proceed with the investigation of the purely inseparable extension

$$
\mathrm{H} \hookrightarrow \mathbb{F}[V] .
$$

We consider the general case of exponent $e \geq 1$. Thus we need to detect $p^{s}$ th powers for $s=1, \ldots, e$. We introduce the following operators for $s \in \mathbb{N}_{0}$ :

$$
\begin{aligned}
& \mathscr{P}^{\Delta_{s, 0}}=\frac{1}{p^{s}} \operatorname{deg}(-) \operatorname{id}(-), \\
& \mathscr{P}^{\Delta_{s, 1}}=\mathscr{P}^{p^{s}}, \\
& \mathscr{P}^{\Delta_{s, i}}=\mathscr{P}^{p^{s} q^{i-1}} \mathscr{P}^{\Delta_{s, i-1}}-\mathscr{P}^{\Delta_{s, i-1}} \mathscr{P}^{p^{s} q^{i-1}} \quad \text { for } i \geq 2 .
\end{aligned}
$$

Remark. Note that for all $s \in \mathbb{N}_{0}$ we have $\mathscr{P}^{\Delta_{s, i}} \in \mathcal{P}^{*}$ whenever $i \neq 0$.

Remark. Note also that the degree of $\mathscr{P}^{\Delta_{s, i}}$ is equal to $q^{i} p^{s}-p^{s}$, for all $i, s \in \mathbb{N}_{0}$. 
Proposition 4.1. The operators $\mathscr{P}^{\Delta_{s, i}}$ satisfy the following properties:

(1) For all $i \in \mathbb{N}$ we have $\mathscr{P}^{\Delta_{s, i}}\left(h^{p}\right)=\left(\mathscr{P}^{\Delta_{s-1, i}}(h)\right)^{p}$ for $h \in \mathrm{H}$ and $s \geq 1$.

(2) For $i \geq 1$ and $k, s \geq 0$ we have

$$
\left[\mathscr{P}^{s} k, \mathscr{P}^{\Delta_{s, i}}\right]=\mathscr{P}^{\Delta_{s, i+1}} \mathscr{P}^{p^{s} k-p^{s} q^{i}} .
$$

(3) For $i, j \geq 1$ and $s \geq 0$ we have

$$
\left[\mathcal{P}^{\Delta_{s, i}}, \mathcal{P}^{\Delta_{s, j}}\right]=0 .
$$

(4) The pth iteration $\mathscr{P}^{\Delta_{s, i}} \ldots \mathscr{P}^{\Delta_{s, i}}=0$ for all $i \geq 1$ and $s \geq 0$.

Proof. $\mathrm{AD}(1)$ : For any $i, j \geq 0$ and any linear form $l$ we have

$$
\mathscr{P}^{i}\left(l^{j}\right)=\left(\begin{array}{l}
j \\
i
\end{array}\right) l^{i q+j-i}
$$

as it can be easily seen by induction. Thus for all $i \geq 0$ we have

$$
\mathscr{P}^{i}\left(l^{p^{s}}\right)=\left(\begin{array}{c}
p^{s} \\
i
\end{array}\right) l^{i q+p^{s}-i} \text {. }
$$

Since $\left(\begin{array}{c}p^{s} \\ i\end{array}\right) \equiv 0(p)$ precisely when $i \notin\left\{p^{s}, 0\right\}$, we have

$$
\mathscr{P}^{i}\left(l^{s^{s}}\right)= \begin{cases}\mathscr{P}^{0}(l) p^{s} & \text { for } i=0, \\ \mathscr{P}^{1}(l)^{p^{s}} & \text { for } i=p^{s} \\ 0 & \text { otherwise. }\end{cases}
$$

Therefore

$$
\mathscr{P}^{i}\left(h^{p^{s}}\right)= \begin{cases}h^{p^{s}} & \text { for } i=0, \\ \mathscr{P}^{k}(h)^{p^{s}} & \text { for } i=k p^{s} \\ 0 & \text { otherwise }\end{cases}
$$

for any $h \in \mathrm{H}$ (cf. page 261 of [6]). Thus

$$
\mathscr{P}^{\Delta_{s, 1}}\left(h^{p}\right)=\mathscr{P}^{p^{s}}\left(h^{p}\right)=\left(\mathscr{P}^{p^{s-1}}(h)\right)^{p}=\left(\mathscr{P}^{\Delta_{s-1,1}}(h)\right)^{p} .
$$

Thus by induction on $i$ we find

$$
\begin{aligned}
\mathscr{P}_{s, i}^{\Delta_{s}}\left(h^{p}\right) & =\mathscr{P}^{s} q^{i-1} \mathscr{P}^{\Delta_{s, i-1}}\left(h^{p}\right)-\mathscr{P}^{\Delta_{s, i-1}} \mathscr{P}^{s} q^{i-1}\left(h^{p}\right) \\
& =\mathscr{P}^{s} q^{i-1}\left(\mathscr{P}^{\Delta_{s-1, i-1}}(h)\right)^{p}-\mathscr{P}^{\Delta_{s, i-1}}\left(\mathscr{P}^{p^{-1}} q^{i-1}(h)\right)^{p} \\
& =\left(\mathscr{P}^{p^{s-1}} q^{i-1} \mathscr{P}^{\Delta_{s-1, i-1}}(h)-\mathscr{P}^{\Delta_{s-1, i-1}} \mathscr{P}^{p^{s-1}} q^{i-1}(h)\right)^{p} \\
& =\left(\mathscr{P}^{\Delta_{s-1, i}}(h)\right)^{p},
\end{aligned}
$$

as claimed.

$\mathrm{AD}(2)$ and (3): The result follows, because it is true for any linear form.

$\mathrm{AD}(4)$ : From the Adem relations it follows that

$$
\mathscr{P}^{p^{s}} \ldots \mathscr{P} p^{s}=0 .
$$

Thus the result follows by induction on $i$ with the help of the commutation rules of (2) (cf. Lemma A.1.1 in [3]).

Define the $\mathrm{H}^{p^{s}}$-module

$$
\operatorname{Der}_{\mathrm{H}, s}=\operatorname{span}_{\mathrm{H}^{p^{s}}}\left\{\mathscr{P}^{\Delta_{s, i}} \mid i \in \mathbb{N}_{0}\right\} .
$$

By definition it follows that $\operatorname{Der}_{\mathrm{H}, 0}=\operatorname{Der}_{\mathrm{H}}$. 
Proposition 4.2. The module $\operatorname{Der}_{\mathrm{H}, s}$ has the following properties:

(1) Der ${ }_{\mathrm{H}, s}$ acts in $\mathrm{H}^{p^{s}}$ as derivations.

(2) For $s, k \geq 0$ we obtain

$$
\left[\mathscr{P}^{p^{s} k}, \mathscr{P}^{\Delta_{s, 0}}\right]=k \mathscr{P}^{k p^{s}} .
$$

(3) If $s \geq 0$, then

$$
\left[\mathscr{P}^{\Delta_{s, i}}, \mathscr{P}^{\Delta_{s, j}}\right]= \begin{cases}\mathscr{P}^{\Delta_{s, i}} & \text { if } i \neq 0 \text { and } j=0, \\ -\mathscr{P}^{\Delta_{s, j}} & \text { if } i=0 \text { and } j \neq 0, \\ 0 & \text { otherwise. }\end{cases}
$$

(4) The pth iteration gives $\mathscr{P}^{\Delta_{s, 0}} \ldots \mathscr{P}^{\Delta_{s, 0}}\left(h^{p^{s}}\right)=\mathscr{P}^{\Delta_{s, 0}}\left(h^{p^{s}}\right)$ for all $s \geq 0$ and $h \in H$.

(5) Let $s \geq 0$. Then $\mathscr{P}^{\Delta_{s, i}}\left(h^{p^{s}}\right)=0 \forall i$ if and only if $h$ is a $p^{s+1}$ st power.

Proof. $\mathrm{AD}(1)$ : Let $h^{p^{s}} \in \mathrm{H}^{p^{s}}$. By Proposition 4.1 Der $\operatorname{Der}_{\mathrm{H}, s}$ acts on $\mathrm{H}^{p^{s}}$ according to the following formulae:

$$
\begin{aligned}
& \mathscr{P}^{\Delta_{s, 0}}\left(h^{p^{s}}\right)=\operatorname{deg}(h) h^{p^{s}}=\mathscr{P}^{\Delta_{0}}(h)^{p^{s}}, \\
& \mathscr{P}^{\Delta_{s, 1}}\left(h^{p^{s}}\right)=\left(\mathscr{P}^{1}(h)\right)^{p^{s}}=\left(\mathscr{P}^{\Delta_{1}}(h)\right)^{p^{s}}, \\
& \mathscr{P}^{\Delta_{s, i}}\left(h^{p^{s}}\right)=\left(\mathscr{P}^{i-1} \mathscr{P}^{\Delta_{i-1}}-\mathscr{P}^{\Delta_{i-1}} \mathscr{P}^{q^{i-1}}(h)\right)^{p^{s}}=\left(\mathscr{P}^{\Delta_{i}}(h)\right)^{p^{s}} .
\end{aligned}
$$

Since taking $p$ th powers is additive in characteristic $p$, this establishes the statement.

$\mathrm{AD}(2)$ : Let $h^{p^{s}} \in \mathrm{H}^{p^{s}}$. We have

$$
\begin{aligned}
{\left[\mathscr{P}^{s^{s} k}, \mathscr{P}^{\Delta_{s, 0}}\right]\left(h^{p^{s}}\right) } & =\mathscr{P}^{p^{s} k} \mathscr{P}^{\Delta_{s, 0}}\left(h^{p^{s}}\right)-\mathscr{P}^{\Delta_{s, 0}} \mathscr{P}^{p^{s} k}\left(h^{p^{s}}\right) \\
& =\operatorname{deg}(h) \mathscr{P}^{p^{s} k}\left(h^{p^{s}}\right)-\mathscr{P}^{\Delta_{s, 0}}\left(\mathscr{P}^{k}(h)\right)^{p^{s}} \\
& =\operatorname{deg}(h) \mathscr{P}^{p^{s} k}\left(h^{p^{s}}\right)-\operatorname{deg}\left(\mathscr{P}^{k}(h)\right) \mathscr{P}^{p^{s} k}\left(h^{p^{s}}\right) \\
& =(\operatorname{deg}(h)-\operatorname{deg}(h)+k-k q) \mathscr{P}^{p^{s} k}\left(h^{p^{s}}\right) \\
& =k \mathscr{P} p^{s} k\left(h^{p^{s}}\right) .
\end{aligned}
$$

$\mathrm{AD}(3):$ Let $h^{p^{s}} \in \mathrm{H}^{p^{s}}$. If $i, j \geq 1$, then

$$
\left[\mathscr{P}^{\Delta_{s, 0}}, \mathscr{P}^{\Delta_{s, j}}\right]=0
$$

by part (3) of Proposition 4.1. Otherwise we have

$$
\begin{aligned}
{\left[\mathscr{P}^{\Delta_{s, 0}}, \mathscr{P}^{\Delta_{s, j}}\right]\left(h^{p^{s}}\right) } & =\mathscr{P}^{\Delta_{s, 0}} \mathscr{P}^{\Delta_{s, j}}\left(h^{p^{s}}\right)-\mathscr{P}^{\Delta_{s, j}} \mathscr{P}^{\Delta_{s, 0}}\left(h^{p^{s}}\right) \\
& =\left(\operatorname{deg}(h)+q^{j}-1\right) \mathscr{P}^{\Delta_{s, j}}\left(h^{p^{s}}\right)-\operatorname{deg}(h) \mathscr{P}^{\Delta_{s, j}}\left(h^{p^{s}}\right) \\
& =-\mathscr{P}^{\Delta_{s, j}}\left(h^{p^{s}}\right) .
\end{aligned}
$$

The relation for $j=0$ can be established in the same way.

$\operatorname{AD}(4)$ : Let $h^{p^{s}} \in \mathrm{H}^{p^{s}}$. Since $\mathscr{P}^{\Delta_{s_{0}}}\left(h^{p^{s}}\right)=\operatorname{deg}(h) h^{p^{s}}$ and $\operatorname{deg}(h)^{p^{s}}=\operatorname{deg}(h)$ the result follows.

$\operatorname{Ad}(5)$ : Let $h^{p^{s}}=\mathrm{H}^{p^{s}}$ and $i \geq 1$. Then

$$
\mathscr{P}^{\Delta_{s, i}}\left(h^{p^{s}}\right)=\left(\mathscr{P}^{\Delta_{i}}(h)\right)^{p^{s}}
$$

and

$$
\mathscr{P}^{\Delta_{s, 0}}\left(h^{p^{s}}\right)=\operatorname{deg}(h) h^{p^{s}}=\mathscr{P}^{\Delta_{0}}(h)^{p^{s}}
$$


are simultaneously zero for all $i$ if and only if

$$
\mathscr{P}^{\Delta_{i}}(h)=0
$$

for all $i \geq 0$, i.e., if and only if $h$ is a $p$ th power; hence precisely when $h^{p^{s}}$ is a $p^{s+1}$ st power.

Thus $\operatorname{Der}_{\mathrm{H}, s}$ is a restricted Lie algebra of derivations acting on $\mathrm{H}^{p^{s}}$ vanishing precisely on the $p^{s+1}$ st powers. We need to have a look at the relation between $\operatorname{Der}_{\mathrm{H}, s}$ and $\operatorname{Der}_{\mathrm{H}}$.

Proposition 4.3. Let $\mathrm{H}$ be an unstable reduced Noetherian algebra over the Steenrod algebra. The action of $\operatorname{Der}_{\mathrm{H}, s}$ on $\mathrm{H}^{p^{s}}$ has the following properties:

(1) For any $d_{s} \in \operatorname{Der}_{\mathrm{H}, s}$ there exists a $d \in \operatorname{Der}_{\mathrm{H}}$ such that

$$
d_{s}\left(h^{p^{s}}\right)=d(h)^{p^{s}} \quad \forall h \in \mathrm{H} .
$$

(2) If there are $m$ derivations in Der $_{\mathrm{H}, s}$ that are linearly dependent, then so are any $m$ derivations.

Proof. $\mathrm{AD}(1)$ : For any

$$
d_{s}=h_{0}^{p^{s}} \mathscr{P}^{\Delta_{s, i_{0}}}+\cdots+h_{l}^{p^{s}} \mathscr{P}^{\Delta_{s, i_{l}}} \in \operatorname{Der}_{\mathrm{H}, s}
$$

we find that

$$
d_{s}=(d)^{p^{s}}=\left(h_{0} \mathscr{P}^{\Delta_{i_{0}}}+\cdots+h_{l} \mathscr{P}^{\Delta_{i_{l}}}\right)^{p^{s}}
$$

by part (1) of Proposition 4.1. By construction $d \in$ Der $_{\mathrm{H}}$.

$\mathrm{AD}(2)$ : Let $d_{s, 1}, \ldots, d_{s, m} \in \operatorname{Der}_{\mathrm{H}, s}$ be linearly dependent. By part (1) we find $d_{1}, \ldots, d_{m} \in \operatorname{Der}_{\mathrm{H}}$ such that $\left(d_{i}(h)\right)^{p^{s}}=d_{s, i}\left(h^{p^{s}}\right)$ for all $h \in \mathrm{H}$. Thus the elements $d_{1}, \ldots, d_{m} \in \operatorname{Der}_{\mathrm{H}}$ are linearly dependent. Therefore any $m$ elements, say $d_{1}^{\prime}, \ldots, d_{m}^{\prime}$, of $\operatorname{Der}_{\mathrm{H}}$ are linearly dependent with a relation

$$
h_{1} d_{1}^{\prime} \cdots+h_{m} d_{m}^{\prime}=0 .
$$

Thus

$$
h_{1}^{p^{s}} d_{s, 1}^{\prime}+\cdots+h_{m}^{p^{s}} d_{s, m}^{\prime}=0
$$

is a relation in $\operatorname{Der}_{\mathrm{H}, s}$.

Thus the minimal $l_{s}$ such that $l_{s}+1$ elements of $\operatorname{Der}_{\mathrm{H}, s}$ are linearly dependent is uniquely defined. We call $l_{s}$ the $\Delta_{s}$-length of $\mathrm{H}^{p^{s}}$, denoted by $\lambda_{\mathrm{H}, s}$ or if no confusion can arise by $\lambda_{s}$. If $\lambda_{s} \in \mathbb{N}_{0}$, we call the algebra $\mathrm{H}^{p^{s}} \Delta_{s}$-finite. Note that by construction $\mathrm{H}$ is $\Delta$-finite if and only if $\mathrm{H}^{p^{s}}$ is $\Delta_{s}$-finite.

Proposition 4.4. Let $\mathrm{H}$ be an unstable reduced $\Delta$-finite algebra over the Steenrod algebra. Let $\lambda$ be its $\Delta$-length and $\lambda_{s}$ the $\Delta_{s}$-length of $\mathrm{H}^{p^{s}}$. Then

(1) $\lambda_{s}=\lambda$.

(2) We have a relation of the form

$$
\mathbf{d}_{s}=\mathbf{d}_{\lambda_{s}, 0}^{p^{s}} \mathscr{P}^{\Delta_{s, 0}}+\cdots+\mathbf{d}_{\lambda_{s}, \lambda_{s}}^{p^{s}} \mathscr{P}^{\Delta_{s, \lambda_{s}}}
$$

on $\mathrm{H}^{p^{s}}$.

Proof. AD(1): Let

$$
d=\mathbf{d}_{\lambda, 0} \mathscr{P}^{\Delta_{0}}+\cdots+(-1)^{\lambda} \mathbf{d}_{\lambda, \lambda} \mathscr{P}^{\Delta_{\lambda}}=\operatorname{Der}_{\mathrm{H}}
$$


be the $\Delta$-relation of $\mathrm{H}$. Then the element $d_{s}\left(h^{p^{s}}\right)=(d(h))^{p^{s}}$ of $\operatorname{Der}_{\mathrm{H}, s}$ vanishes on $\mathrm{H}^{p^{s}}$. Thus $\lambda_{s} \leq \lambda$. Conversely, if

$$
d_{s}=h_{0}^{p^{s}} \mathscr{P}^{\Delta_{s, 0}}+\cdots+h_{\lambda_{s}}^{p^{s}} \mathscr{P}^{\Delta_{s, \lambda_{s}}} \in \operatorname{Der}_{\mathrm{H}, s}
$$

is a $\Delta_{s}$-relation of $\mathrm{H}^{p^{s}}$, then the element

$$
d=h_{0} \mathscr{P}^{\Delta_{0}}+\cdots+h_{\lambda_{s}} \mathscr{P}^{\Delta_{\lambda_{s}}} \in \operatorname{Der}_{\mathrm{H}}
$$

vanishes on $\mathrm{H}$, by part (1) of Proposition 4.3. Thus, also, $\lambda \leq \lambda_{s}$.

$\operatorname{AD}(2)$ : $\mathrm{By}(1) \lambda_{s}$ is the $\Delta$-length of $\mathrm{H}$. Therefore

$$
\mathbf{d}=\mathbf{d}_{\lambda_{s}, 0} \mathscr{P}^{\Delta_{0}}+\cdots+\mathbf{d}_{\lambda_{s}, \lambda_{s}} \mathscr{P}^{\Delta_{\lambda_{s}}}
$$

is the $\Delta$-relation on $\mathrm{H}$. Thus the result follows by part (1) of Proposition 4.3.

We call the relation $\mathbf{d}_{s}$ of part (2) of this result the $\Delta_{s}$-relation of $\mathrm{H}^{p_{s}}$.

Let $\mathrm{H}^{p^{s}} \hookrightarrow \mathbb{F}[V]^{p^{s}}$ with $\Delta_{s}$-length $\lambda_{s}$. Then $\mathrm{H}=\mathrm{H}_{s}^{p^{s}} \hookrightarrow \mathbb{F}[V]$, since $\mathrm{H}_{s}^{p^{s}}$ arises from $\mathrm{H}^{p^{s}}$ by adjoining all $p^{s}$ th roots. Let $\mathrm{H}$ have $\Delta$-length $\lambda$. Thus $\lambda=\lambda_{s} \leq n$ by Proposition 4.4, part (1).

Proposition 4.5. For $s \geq 0$ we have

$$
\left(\mathcal{C}_{\operatorname{Der}_{\mathrm{H}}}(\mathrm{H})\right)^{p^{s}}=\mathcal{C}_{\operatorname{Der}_{\mathrm{H}, s}}\left(\mathrm{H}^{p^{s}}\right) \subseteq \mathcal{C}_{\text {Der }_{\mathrm{H}}}(\mathrm{H}) .
$$

Proof. If $h^{p^{s}} \in \mathrm{H}^{p^{s}}$ is a $\operatorname{Der}_{\mathrm{H}, s^{-}}$constant, then $h$ is a $p^{s+1}$ st power by part (5) of Proposition 4.2. Thus there exists an element $k \in \mathrm{H}_{1}^{p^{s}}=\mathrm{H}^{p^{s-1}}$ such that $k^{p}=h^{p^{s}}$. Hence $h^{p^{s}}=k^{p} \in \mathrm{H}_{1}^{p^{s}} \subseteq \mathrm{H}$ is a $p$ th power, i.e., a Der $\mathrm{H}_{\text {-constant. }}$

In order to prove the equality, let $h \in \mathrm{H}$ be a Der $\mathrm{H}^{-c o n s t a n t, ~ i . e ., ~} d(h)=0$ for all $d \in \operatorname{Der}_{\mathrm{H}}$. For any $d_{s} \in \operatorname{Der}_{\mathrm{H}, s}$ there is an element $d \in \operatorname{Der}_{\mathrm{H}}$ such that $d_{s}\left(h^{p^{s}}\right)=$ $(d(h))^{p^{s}}=0$ by part (1) of Proposition 4.3. Thus $\left(\mathcal{C}_{\text {Der }_{\mathrm{H}}}(\mathrm{H})\right)^{p^{s}} \subseteq \mathcal{C}_{\text {Der }_{\mathrm{H}, s}}\left(\mathrm{H}^{p^{s}}\right)$. Conversely, if $h^{p^{s}} \in \mathcal{C}_{\text {Der }_{\mathrm{H}, s}}\left(\mathrm{H}^{p^{s}}\right)$, then $h \in \mathcal{C}_{\text {Der }_{\mathrm{H}}}(\mathrm{H})$, by what we have proven so far. Thus $h^{p^{s}} \in\left(\mathcal{C}_{\text {DerH }_{\mathrm{H}}}(\mathrm{H})\right)^{p^{s}}$ as desired.

Remark. We note that the preceding results imply that

$$
\operatorname{dim}\left(\operatorname{Der}_{\mathbb{F}[V], s}\right)=\operatorname{dim}\left(\operatorname{Der}_{\mathbb{F}[V]}\right)=\operatorname{dim}_{\mathbb{F}}(V)=n .
$$

Remark. In Section 3 we defined $\operatorname{Der}_{\mathbb{H}}$ for $\mathbb{H}=F F(\mathrm{H})$ for integral domains $\mathrm{H}$. In the same way we define

$$
\operatorname{Der}_{\mathbb{H}, s}=\operatorname{span}_{\mathbb{H} \rho^{s}}\left\{\mathscr{P}^{\Delta_{i, s}} \mid i \in \mathbb{N}_{0}\right\} .
$$

We obtain analogously to Proposition 3.5 that $\operatorname{dim}\left(\operatorname{Der}_{\mathbb{H}, s}\right)=\operatorname{dim}\left(\operatorname{Der}_{\mathrm{H}, s}\right)$. Hence, $\lambda_{\mathbb{H}, s}=\lambda_{\mathrm{H}, s}$ and $\mathbf{d}_{\mathbb{H}, s}=\mathbf{d}_{\mathrm{H}, s}$. Therefore Der $\operatorname{De}_{\mathbb{H}, s}$ and $\operatorname{Der}_{\mathrm{H}, s}$ have basis $\left\{\mathscr{P}_{s, 0}, \ldots\right.$, $\mathscr{P}^{\Delta_{s, \lambda_{s}}}$, where $\lambda_{s}=\lambda_{\mathbb{H}, s}=\lambda_{\mathrm{H}, s}$.

Corollary 4.6. The $\Delta_{s}$-length of $\mathbb{F}[V]^{p^{s}}$, resp. $\mathbb{F}(V)^{p^{s}}$, is equal to the dimension of $V$. Moreover, for $\mathrm{D}_{s}=0$

$$
\mathbb{F}[V]^{p^{s}}=\mathcal{C}_{\mathrm{D}_{s}}\left(\mathbb{F}[V]^{p^{s}}\right), \quad \text { resp. } \mathbb{F}(V)^{p^{s}}=\mathcal{C}_{\mathrm{D}_{s}}\left(\mathbb{F}(V)^{p^{s}}\right) .
$$

Finally, the $\Delta_{s}$-length of $\mathbb{F}[V]^{p^{s+1}}$, resp. $\mathbb{F}(V)^{p^{s+1}}$, is zero, and

$$
\mathbb{F}[V]^{p^{s+1}}=\mathcal{C}_{\operatorname{Der}_{\mathbb{F}[V], s}}\left(\mathbb{F}[V]^{p^{s}}\right), \quad \text { resp. } \mathbb{F}(V)^{p^{s+1}}=\mathcal{C}_{\operatorname{Der}_{\mathbb{F}(V), s}}\left(\mathbb{F}(V)^{p^{s}}\right) .
$$

Proof. The first statement follows from Lemma 3.8 and Proposition 4.5 The second statement follows from Corollaries 3.2 and 3.9 and Proposition 4.5 . 
We need a generalization of Lemma 3.7

Lemma 4.7. Let $\mathrm{K}^{p^{s}} \subseteq \mathrm{H}^{p^{s}}$ be unstable reduced Noetherian algebras over the Steenrod algebra. Denote by $\lambda_{\mathrm{K}, s}$, resp. $\lambda_{\mathrm{H}, s}$, the $\Delta_{s}$-length of $\mathrm{K}^{p^{s}}$, resp. $\mathrm{H}^{p^{s}}$. Then $\lambda_{\mathrm{K}, s} \leq \lambda_{\mathrm{H}, s}$.

Proof. The proof works just as the one of Lemma 3.7 .

Proposition 4.8. Let $t>s$ and $W \leq V$. The $\Delta_{s}$-length of $\mathbb{F}[W]^{p^{s}} \otimes \mathbb{F}[V / W]^{p^{t}}$ as well as of $\mathbb{F}(W)^{p^{s}} \otimes \mathbb{F}(V / W)^{p^{t}}$, is equal to $\lambda_{s}=\operatorname{dim}_{\mathbb{F}}(W)$. Moreover, let $\mathrm{H}^{p^{s}} \hookrightarrow$ $\mathbb{F}[V]^{p^{s}}$. Then the $\Delta_{s}$-length of $\mathrm{H}^{p^{s}}$ is at most $l$ if and only if

$$
\mathrm{H}^{p^{s}} \subseteq \mathbb{F}[W]^{p^{s}} \otimes \mathbb{F}[V / W]^{p^{t}},
$$

where $\operatorname{dim}_{\mathbb{F}}(W)=l$ and $t>s$.

Proof. The first statement follows from Proposition 4.4, Lemma 3.8, and Corollary [3.9, If $\mathrm{H}^{p^{s}} \subseteq \mathbb{F}[W]^{p^{s}} \otimes \mathbb{F}[V / W]^{p^{t}}$, then by Lemma 4.7 its $\Delta_{s}$-length is at most the $\Delta_{s}$-length of $\mathbb{F}[W]^{p^{s}} \otimes \mathbb{F}[V / W]^{p^{t}}$, which is $l$ by what we proved so far.

Conversely, let the $\Delta_{s}$-length of $\mathrm{H}^{p^{s}}$ be $\lambda_{s} \leq l$. Then $\mathrm{H}$ has $\Delta$-length $\lambda=\lambda_{s}$ by Proposition 4.4. Thus its $\Delta$-relation is

$$
\mathbf{d}=\mathbf{d}_{\lambda, 0} \mathscr{P}^{\Delta_{0}}+\cdots \mathbf{d}_{\lambda, \lambda} \mathscr{P}^{\Delta_{\lambda}}
$$

and therefore the Dickson algebra of dimension $\lambda$ is contained in $\mathrm{H}$.

$$
\mathcal{D}(\lambda) \hookrightarrow \mathrm{H} \hookrightarrow \mathbb{F}[U] \otimes \mathbb{F}[V / U]
$$

for some $U \leq V$ with $\operatorname{dim}(U)=\lambda$. But $\mathbb{F}[U] \otimes \mathbb{F}[V / U]^{p}$ is the largest subalgebra of $\mathbb{F}[V]$ with $\Delta$-length $\lambda$ by Corollary 3.14 . Thus

$$
\mathrm{H} \hookrightarrow \mathbb{F}[U] \otimes \mathbb{F}[V / U]^{p} \hookrightarrow \mathbb{F}[W] \otimes \mathbb{F}[V / W]^{p}
$$

and the result follows.

Lemma 4.9. We have

$$
F F\left(\mathcal{C}_{\operatorname{Der}_{\mathrm{H}, s}}\left(\mathrm{H}^{p^{s}}\right)\right)=\mathcal{C}_{\operatorname{Der}_{\mathbb{H}, s}}\left(\mathbb{H}^{p^{s}}\right) .
$$

Proof. This follows from Propositions 4.5 and 2.3.

In order to be able to treat the general case, we need another preliminary result.

Proposition 4.10. Let $V=W_{0} \oplus \cdots \oplus W_{e}$ be a vector space decomposition. Consider the purely inseparable extension $\mathrm{H}^{p^{s_{0}}} \hookrightarrow \mathbb{F}\left[W_{0}\right]^{p^{s_{0}}} \otimes \cdots \otimes \mathbb{F}\left[W_{e}\right]^{s^{s_{e}}}$. Let $s_{0}<\cdots<s_{e}$. Let $\mathrm{H}^{p^{s_{0}}}$ be an integrally closed unstable algebra over the Steenrod algebra. Then the $\Delta_{s_{0}}$-length of $\mathrm{H}^{p^{s_{0}}}$ is $\lambda_{s_{0}}$ if and only if

$$
\mathbb{F}\left[U_{0}\right]^{p^{s_{0}}} \hookrightarrow \mathbb{H}^{p^{s_{0}}} \hookrightarrow \mathbb{F}[U-0]^{p^{s_{0}}} \otimes \mathbb{F}\left[W_{0} / U_{0}\right]^{p^{s_{0}+1}} \otimes \mathbb{F}\left[W_{1}\right]^{p^{s_{1}}} \otimes \cdots \otimes \mathbb{F}\left[W_{e}\right]^{p^{s_{e}}}
$$

for $\operatorname{dim}_{\mathbb{F}}\left(U_{0}\right)=\lambda_{s_{0}}, U_{0} \leq W_{0}$ and $U_{0}$ maximal with respect to this property.

Proof. Let the $\Delta_{s_{0}}$-length of $\mathrm{H}^{p_{s_{0}}}$ be $\lambda_{s_{0}}$. Note that $\lambda_{s_{0}} \leq \operatorname{dim}_{\mathbb{F}}\left(W_{0}\right)$ by Lemma4.7. The $\Delta$-length of $\mathrm{H}$ is also $\lambda_{s_{0}}$ by part (1) of Proposition 4.4. Moreover

$$
\mathrm{H} \hookrightarrow \mathbb{F}\left[W_{0}\right] \otimes \mathbb{F}\left[W_{1}\right]^{p^{s_{1}-s_{0}}} \otimes \cdots \otimes \mathbb{F}\left[W_{e}\right]^{p^{s_{e}-s_{0}}} .
$$


Let $\mathbf{d}$ be the $\Delta$-relation of $H$. Then $\mathbf{d}$ is also the $\Delta$-relation of its field of fractions, $\mathbb{H}$. Thus by construction we find for $\mathrm{D}=\mathrm{D}_{\mathbb{H}} \subseteq \operatorname{Der}_{\mathbb{H}}$

$$
\begin{aligned}
\mathbb{H} \hookrightarrow \mathcal{C}_{\mathrm{D}}\left(\mathbb{F}\left(W_{0}\right) \otimes \mathbb{F}\left(W_{1}\right)^{p^{s_{1}-s_{0}}} \otimes \cdots \otimes \mathbb{F}\left(W_{e}\right)^{p^{s_{e}-s_{0}}}\right) \\
\quad=\mathbb{F}\left(U_{0}\right) \otimes \mathbb{F}\left(W_{0} / U_{0}\right)^{p} \otimes \mathbb{F}\left(W_{1}\right)^{p^{s_{1}-s_{0}}} \otimes \cdots \otimes \mathbb{F}\left(W_{e}\right)^{p^{s_{e}-s_{0}}}
\end{aligned}
$$

for some $U_{0} \leq W_{0}$ of dimension $\lambda_{s_{0}}$ by Corollary 3.9. Let $U$ be maximal with this property. The extension

$$
\mathbb{F}\left(U_{0}\right) \cap \mathbb{H} \hookrightarrow \mathbb{F}\left(U_{0}\right)
$$

is purely inseparable, since

$$
\mathbb{H} \hookrightarrow \mathbb{F}\left(U_{0}\right) \otimes \mathbb{F}\left(W_{0} / U_{0}\right)^{p} \otimes \mathbb{F}\left(W_{1}\right)^{p^{s_{1}-s_{0}}} \otimes \cdots \otimes \mathbb{F}\left(W_{e}\right)^{p^{s_{e}-s_{0}}}
$$

is purely inseparable and all elements in $\mathbb{F}\left(U_{0}\right) \otimes \mathbb{F}\left(W_{0} / U_{0}\right)^{p} \otimes \mathbb{F}\left(W_{1}\right)^{p^{s_{1}-s_{0}}} \otimes \cdots \otimes$ $\mathbb{F}\left(W_{e}\right)^{p^{s_{e}-s_{0}}}$ that are algebraic over $\mathbb{F}\left(U_{0}\right)$ are inseparable. By maximality of $\lambda_{s_{0}}$ all elements in $\mathbb{F}\left(U_{0}\right)$ are separable over $\mathbb{H}$. Thus

$$
\mathbb{F}\left(U_{0}\right) \cap \mathbb{H} \hookrightarrow \mathbb{F}\left(U_{0}\right)
$$

is also separable. Hence

$$
\mathbb{F}\left(U_{0}\right)=\mathbb{F}\left(U_{0}\right) \cap \mathbb{H} \hookrightarrow \mathbb{H} .
$$

Therefore

$$
\mathbb{F}\left[U_{0}\right]^{p^{s_{0}}} \hookrightarrow \mathrm{H}^{p^{s_{0}}} \hookrightarrow \mathbb{F}\left[U_{0}\right]^{p^{s_{0}}} \otimes \mathbb{F}\left[W_{0} / U_{0}\right]^{p^{s_{0}+1}} \otimes \cdots \otimes \mathbb{F}\left[W_{e}\right]^{p^{s_{e}}}
$$

as desired.

To prove the converse, assume that there exists a vector space $U_{0} \leq W_{0}$ of $\operatorname{dim}_{\mathbb{F}}\left(U_{0}\right)=\lambda_{s_{0}}$ such that

$$
\mathbb{F}\left[U_{0}\right]^{p^{s_{0}}} \hookrightarrow \mathrm{H}^{p^{s_{0}}} \hookrightarrow \mathbb{F}\left[U_{0}\right]^{p^{s_{0}}} \otimes \mathbb{F}\left[W_{0} / U_{0}\right]^{p^{s_{0}+1}} \otimes \cdots \otimes \mathbb{F}\left[W_{e}\right]^{s^{s_{e}}} .
$$

Assume furthermore that $U_{0}$ is maximal with this property. Then the $\Delta_{s_{0}}$-length of $\mathbb{F}\left[U_{0}\right]^{s_{0}}$ is $\lambda_{s, 0}$ by Corollary 4.6. Equally, the $\Delta_{s_{0}}$-length of $\mathbb{F}\left[U_{0}\right]^{p_{0}} \otimes \mathbb{F}\left[W_{0} / U_{0}\right]^{p^{s_{0}+1}}$ $\otimes \cdots \otimes \mathbb{F}\left[W_{e}\right]^{p^{s_{e}}}$ is $\lambda_{s_{0}}$ by Proposition 4.8. Therefore the $\Delta_{s_{0}}$-length of $H^{p^{s_{0}}}$ is $\lambda_{s_{0}}$ by Lemma 4.7 .

Remark. Note that any element in $\mathrm{H}^{p^{s_{0}}}$ that is algebraic over $\mathbb{F}\left[U_{0}\right]^{p^{s_{0}}}$ is separable over $\mathbb{F}\left[U_{0}\right]^{p^{s_{0}}}$.

Theorem 4.11. Let $V=W_{0} \oplus \cdots \oplus W_{e}$ be a vector space decomposition. Consider the purely inseparable extension $\mathrm{H} \hookrightarrow \mathbb{F}\left[W_{0}\right] \otimes \mathbb{F}\left[W_{1}\right]^{p} \otimes \cdots \otimes \mathbb{F}\left[W_{e}\right]^{p^{e}}$ of exponent one. Let $\mathrm{H}$ be integrally closed. Then $\mathrm{H}$ is an unstable algebra over the Steenrod algebra if and only if

$$
\mathrm{H}=\mathbb{F}\left[U_{0}\right] \otimes \mathbb{F}\left[U_{1}\right]^{p} \otimes \cdots \otimes \mathbb{F}\left[U_{e+1}\right]^{p^{e+1}}
$$

for some vector space decomposition

$$
V=U_{0} \oplus \cdots \oplus U_{e+1}
$$

with

$$
U_{0} \oplus \cdots \oplus U_{i} \leq W_{0} \oplus \cdots \oplus W_{i}
$$

and $\operatorname{dim}\left(U_{0} \oplus \cdots \oplus U_{i}\right)$ is the $\Delta$-length of $\mathrm{H}_{i}, i=0, \ldots, e+1$. 
Proof. The "if" part of the statement is clear by Proposition 4.10, We need to prove the "only if" part.

Let $\mathbf{d} \in \operatorname{Der}_{\mathrm{H}}$ be the $\Delta$-relation on $\mathrm{H}$ of length $\lambda_{0}$. Then $\mathbb{F}\left[U_{0}\right] \hookrightarrow \mathrm{H}$ for some vector space $U_{0}$ of dimension $\lambda_{0}$ by Proposition 4.10. Hence $\lambda_{0} \leq \operatorname{dim}\left(W_{0}\right)$, $U_{0} \leq W_{0}$, and we have

$$
\mathbb{F}\left[U_{0}\right] \hookrightarrow \mathrm{H} \hookrightarrow \mathbb{F}\left[W_{0}\right] \otimes \mathbb{F}\left[W_{1}\right]^{p} \otimes \cdots \otimes \mathbb{F}\left[W_{e}\right]^{p^{e}} .
$$

We consider the chain

$$
\begin{aligned}
\mathbb{F}\left[U_{0}\right]_{1} \hookrightarrow \mathrm{H}_{1} \hookrightarrow\left(\mathbb{F}\left[W_{0}\right] \otimes \mathbb{F}\left[W_{1}\right]^{p} \otimes\right. & \left.\cdots \otimes \mathbb{F}\left[W_{e}\right]^{p^{e}}\right)_{1} \\
& =\mathbb{F}\left[W_{0} \oplus W_{1}\right] \otimes \mathbb{F}\left[W_{2}\right]^{p} \otimes \cdots \otimes \mathbb{F}\left[W_{e}\right]^{p^{e-1}} .
\end{aligned}
$$

By Proposition 4.10 the $\Delta$-length of $\mathrm{H}_{1}$ is at most the dimension of $W_{0} \oplus W_{1}$ and

$$
\mathbb{F}\left[U_{0}\right] \otimes \mathbb{F}\left[U_{1}\right] \hookrightarrow \mathbb{H}_{1}
$$

for a suitable $U_{0} \oplus U_{1} \leq W_{0} \oplus W_{1}$. Since $\mathbb{F}\left[U_{0}\right] \hookrightarrow \mathrm{H}_{1}$, and $U_{0}$ is the maximal vector subspace with this property, we have

$$
\mathbb{F}\left[U_{0}\right] \otimes \mathbb{F}\left[U_{1}\right]^{p} \hookrightarrow \mathrm{H} .
$$

Proceeding inductively gives an extension

$$
\mathbb{F}\left[U_{0}\right] \otimes \mathbb{F}\left[U_{1}\right]^{p} \otimes \cdots \otimes \mathbb{F}\left[U_{e+1}\right]^{p^{e+1}} \hookrightarrow \mathrm{H},
$$

which is separable, because it is algebraic (cf. the remark after Proposition 4.10). This extension is also purely inseparable because

$$
\mathbb{F}\left[U_{0}\right] \otimes \mathbb{F}\left[U_{1}\right]^{p} \otimes \cdots \otimes \mathbb{F}\left[U_{e+1}\right]^{p^{e+1}} \hookrightarrow \mathbb{F}\left[W_{0}\right] \otimes \mathbb{F}\left[W_{1}\right]^{p} \otimes \cdots \otimes \mathbb{F}\left[W_{e+1}\right]^{p^{e+1}}
$$

is purely inseparable. Thus

$$
\mathrm{H}=\mathbb{F}\left[U_{0}\right] \otimes \mathbb{F}\left[U_{1}\right]^{p} \otimes \cdots \otimes \mathbb{F}\left[U_{e+1}\right]^{p^{e+1}}
$$

as desired.

Corollary 4.12. Let $V=W_{0} \oplus \cdots \oplus W_{e}$ be a vector space decomposition. Let $\mathbb{H} \hookrightarrow \mathbb{F}\left(W_{0}\right) \otimes \mathbb{F}\left(W_{1}\right)^{p} \otimes \cdots \otimes \mathbb{F}\left(W_{e}\right)^{p^{e}}$ be a purely inseparable extension of exponent one. Then $\mathbb{H}$ is a field over the Steenrod algebra if and only if

$$
\mathbb{H}=\mathbb{F}\left(U_{0}\right) \otimes \mathbb{F}\left(U_{1}\right)^{p} \otimes \cdots \otimes \mathbb{F}\left(U_{e+1}\right)^{p^{e+1}}
$$

for some vector space decomposition

$$
V=U_{0} \oplus \cdots \oplus U_{e+1}
$$

with

$$
U_{0} \oplus \cdots \oplus U_{i} \leq W_{0} \oplus \cdots \oplus W_{i}
$$

and $\operatorname{dim}\left(U_{0} \oplus \cdots \oplus U_{i}\right)$ is the $\Delta$-length of $\mathbb{H}_{i}, i=0, \ldots, e+1$.

Proof. Since

$$
\mathcal{U} n(\mathbb{H}) \hookrightarrow \mathbb{F}\left[U_{0}\right] \otimes \mathbb{F}\left[U_{1}\right]^{p} \otimes \cdots \otimes \mathbb{F}\left[U_{e}\right]^{p^{e}}
$$

is integrally closed and $F F(\mathcal{U} n(\mathbb{H}))=\mathbb{H}$, the result follows from Theorem4.11, 
Theorem 4.13. $\mathbb{H} \hookrightarrow \mathbb{F}(V)$ is a purely inseparable extension of exponent e of fields over the Steenrod algebra if and only if

$$
\mathbb{H}=\mathbb{F}\left(W_{0}\right) \otimes \mathbb{F}\left(W_{1}\right)^{p} \otimes \cdots \otimes \mathbb{F}\left(W_{e}\right)^{p^{e}}
$$

for some vector space decomposition

$$
V=W_{0} \oplus \cdots \oplus W_{e},
$$

where $\operatorname{dim}\left(W_{0} \oplus \cdots \oplus W_{i}\right)$ is the $\Delta$-length of $\mathbb{H}_{i}, i=0, \ldots$, e.

Proof. The "if" part is clear by Corollary 4.12, We show the "only if" part.

We proceed by induction on $e$. The case $e=1$ has been treated in Theorem4.11. Thus assume that $e>1$.

We have a chain of purely inseparable extensions of exponent one

$$
\mathbb{H}=\mathbb{H}_{0} \hookrightarrow \mathbb{H}_{1} \hookrightarrow \cdots \hookrightarrow \mathbb{H}_{e}=\mathbb{F}(V)
$$

which is obtained by adjoining successively $p$ th roots. Note that all $\mathbb{H}_{i}$ 's are fields over the Steenrod algebra.

By the induction hypothesis we can assume that

$$
\mathbb{H} \hookrightarrow \mathbb{H}_{1}=\mathbb{F}\left(W_{0}\right) \otimes \mathbb{F}\left(W_{1}\right)^{p} \otimes \cdots \otimes \mathbb{F}\left(W_{e-1}\right)^{p^{e-1}}
$$

for a vector space decomposition

$$
V=W_{0} \oplus \cdots \oplus W_{e-1} .
$$

By Corollary 4.12 we are done.

At the level of algebras we obtain the following result as an obvious corollary.

Corollary 4.14. Let $\mathrm{H}$ be integrally closed. Let $\mathrm{H} \hookrightarrow \mathbb{F}[V]$ be a purely inseparable extension of exponent $e$. Then $\mathrm{H}$ is an algebra over the Steenrod algebra if and only if

$$
\mathrm{H}=\mathbb{F}\left[W_{0}\right] \otimes \mathbb{F}\left[W_{1}\right]^{p} \otimes \cdots \otimes \mathbb{F}\left[W_{e}^{p^{e}}\right]
$$

for some vector space decomposition

$$
V=W_{0} \oplus \cdots \oplus W_{e},
$$

where $\operatorname{dim}\left(W_{0} \oplus \cdots \oplus W_{i}\right)$ is the $\Delta$-length of $\mathrm{H}_{i}, i=0, \ldots, e$.

Remark. Note that Corollary 4.14 has been proven in Theorem 7.2.2 of 3 as well as in [7, Theorem II, without, however, the precise statement on the dimension of $W_{0} \oplus \cdots \oplus W_{i}$.

\section{Purely inseparable extensions, the general Case}

Let $\mathrm{H}$ be an unstable Noetherian integral domain over the Steenrod algebra. Assume that the canonical inclusion

$$
\mathrm{H} \hookrightarrow \sqrt[\mathcal{P} *]{\mathrm{H}}
$$

is purely inseparable of exponent $e$.

If $\mathrm{H}$ is integrally closed, then so is $\sqrt[\mathcal{P *}]{\mathrm{H}}$ by part (3) of Proposition 2.1, Then

$$
\sqrt[\mathcal{P}^{*}]{\mathrm{H}} \hookrightarrow \mathbb{F}[V]
$$

is a Galois extension with Galois group $G \leq \mathrm{GL}(n, \mathbb{F})$, where $n$ is the Krull dimension of $\mathrm{H}$ (see the Galois Embedding Theorem, Theorem 7.1.1 in [3]). Thus

$$
\sqrt[\mathcal{P} *]{\mathrm{H}}=\mathbb{F}[V]^{G} .
$$


On the other hand we can take the separable closure first: The separable closure of $\mathrm{H} \hookrightarrow \mathbb{F}[V]$ denoted by $\overline{\mathrm{H}}^{s e p}$ is again an unstable algebra over the Steenrod algebra by the Separable Extension Lemma (Proposition 2.2.2 in [3]), since $\overline{\mathrm{H}}^{\text {sep }}=\mathcal{U n}_{n}\left(\overline{\mathbb{H}}^{\text {sep }}\right.$ ). Thus we obtain a purely inseparable extension of exponent $e$

$$
\overline{\mathrm{H}}^{\text {sep }} \hookrightarrow \mathbb{F}[V] .
$$

Therefore, by Corollary 4.14

$$
\overline{\mathrm{H}}^{\text {sep }}=\mathbb{F}\left[W_{0}\right] \otimes \cdots \otimes \mathbb{F}\left[W_{e}\right]^{p^{e}},
$$

for some vector space decomposition $V=W_{0} \oplus \cdots \oplus W_{e}$.

We need a technical lemma.

Lemma 5.1. Let $\mathrm{H}$ be an unstable Noetherian integral domain over the Steenrod algebra. Then for all $i \in \mathbb{N}_{0}$ we have

$$
\left(\overline{\mathrm{H}}^{\text {sep }}\right)_{i}={\overline{\left(\mathrm{H}_{i}\right)}}^{\text {sep }} .
$$

Proof. By induction on $i$, we need to prove the statement only for $i=1$. By assumption we have the diagram

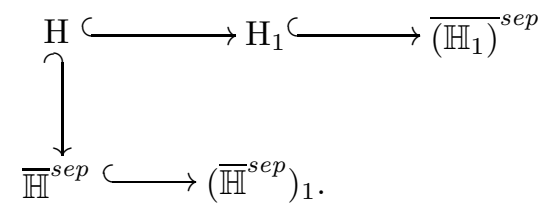

If $h \in \mathrm{H}_{1}$, then $h^{p} \in \mathrm{H} \subseteq \overline{\mathrm{H}}^{s e p}$. Thus $h \in\left(\overline{\mathrm{H}}^{s e p}\right)_{1}$. Thus

$$
\mathrm{H}_{1} \hookrightarrow\left(\overline{\mathrm{H}}^{\text {sep }}\right)_{1} \text {. }
$$

We note that $\overline{\mathrm{H}}^{s e p}=\mathbb{F}\left[W_{0}\right] \otimes \cdots \otimes \mathbb{F}\left[W_{e}\right]^{p^{e}}$ by Corollary 4.14, Therefore

$$
\left(\overline{\mathrm{H}}^{s e p}\right)_{1}=\mathbb{F}\left[W_{0} \oplus W_{1}\right] \otimes \mathbb{F}\left[W_{2}\right]^{p} \otimes \cdots \otimes \mathbb{F}\left[W_{e}\right]^{p^{e-1}} .
$$

Hence $\left(\overline{\mathrm{H}}^{s e p}\right)_{1}$ is separably closed, and thus

$$
{\overline{\left(\mathrm{H}_{1}\right)}}^{\mathrm{sep}} \hookrightarrow\left(\overline{\mathrm{H}}^{\mathrm{sep}}\right)_{1} .
$$

Moreover, this extension is by the universal property of the separable closure purely inseparable. Next we show that this extension has exponent at most one. To this end, take $h \in\left(\overline{\mathrm{H}}^{s e p}\right)_{1}$. Then $h^{p} \in \overline{\mathrm{H}}^{s e p}$ is separable over $\mathrm{H}$, hence over $\mathrm{H}_{1}$. Therefore $h^{p} \in{\overline{\left(\mathrm{H}_{1}\right)}}^{\text {sep }}$.

Denote the inseparable closure of $\mathrm{H}_{1}$ inside $\left(\overline{\mathrm{H}}^{s e p}\right)_{1}$ by $\mathrm{K}$. Then $\mathrm{H}_{1} \hookrightarrow \mathrm{K}$ has

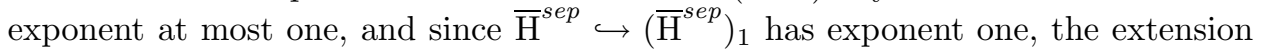
$\mathrm{H} \hookrightarrow \mathrm{K}$ also has exponent at most one. Since $\mathrm{H}_{1}$ is the largest algebra such that $\mathrm{H} \hookrightarrow \mathrm{K}$ has exponent one, we have that $\mathrm{H}_{1}=\mathrm{K}$ and $\mathrm{H}_{1} \hookrightarrow\left(\overline{\mathrm{H}}^{s e p}\right)_{1}$ is separable.

Therefore, ${\overline{\left(\mathrm{H}_{1}\right)}}^{s e p} \hookrightarrow\left(\overline{\mathrm{H}}^{s e p}\right)_{1}$ is also separable. Since we already saw that this extension is purely inseparable, this means that

$$
\left.(\overline{\mathrm{H}})^{s e p}\right)_{1}={\overline{\left(\mathrm{H}_{1}\right)}}^{\text {sep }}
$$

as claimed.

So, in what follows we can write ${\overline{\mathrm{H}_{1}}}^{s e p}$ for ${\overline{\left(\mathrm{H}_{1}\right)}}^{\text {sep }}=\left(\overline{\mathrm{H}}^{s e p}\right)_{1}$ without ambiguity. 
Theorem 5.2. Let $\mathrm{H}$ be an integrally closed unstable Noetherian integral domain over the Steenrod algebra of Krull dimension $n . \quad$ Set $\operatorname{dim}_{\mathbb{F}}(V)=n$. Let $V=$ $W_{0} \oplus \cdots \oplus W_{e}$, and let

$$
\overline{\mathrm{H}}^{\text {sep }}=\mathbb{F}\left[W_{0}\right] \otimes \cdots \otimes \mathbb{F}\left[W_{e}\right]^{p^{e}} .
$$

Then there exists a group $G \leq \mathrm{GL}(V)$ acting on the flags $W_{0} \oplus \cdots \oplus W_{i}$ for $i=$ $0, \ldots$, e such that $\sqrt[\mathcal{P} *]{\mathrm{H}}=\mathbb{F}[V]^{G}$ and

$$
\mathrm{H}=\left(\mathbb{F}\left[W_{0}\right] \otimes \cdots \otimes \mathbb{F}\left[W_{e}\right]^{p^{e}}\right)^{G} .
$$

Furthermore, $\operatorname{dim}_{\mathbb{F}}\left(W_{0} \oplus \cdots \oplus W_{i}\right)$ is the $\Delta$-length of $\mathrm{H}_{i}$.

Proof. By assumption we have a diagram

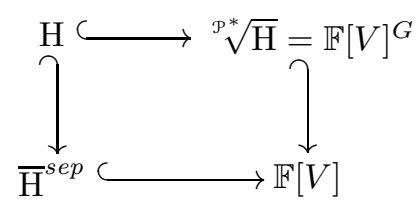

where the horizontal extensions are purely inseparable and the vertical are separable. Recall that

$$
\overline{\mathrm{H}}^{\text {sep }}=\mathbb{F}\left[W_{0}\right] \otimes \cdots \otimes \mathbb{F}\left[W_{e}\right]^{p^{e}}
$$

where $\operatorname{dim}_{\mathbb{F}}\left(W_{0} \oplus \cdots \oplus W_{i}\right)$ is the $\Delta$-length of $\overline{\mathrm{H}}_{i}^{\text {sep }}$ by Corollary 4.14. Consider the corresponding diagram of the respective field of fractions

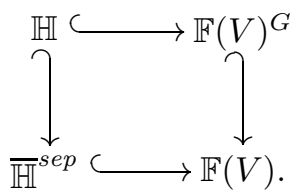

Recall from the Imbedding Theorem (Theorem 8.1.5 in [3]) that $\mathrm{H}$, and hence $\mathbb{H}$, contains a fractal of the Dickson algebra in dimension $n=\operatorname{dim}_{\mathbb{F}}(V)$. Thus

$$
\mathcal{D}(n)^{q^{s}} \hookrightarrow \mathrm{H} \hookrightarrow \mathbb{H} \hookrightarrow \mathbb{F}(V)
$$

for some $s \in \mathrm{N}_{0}$. Therefore, the polynomial

$$
\Delta(X)=\prod_{l \in V^{*}}(X-l)^{q^{s}}=\mathbf{d}_{n, 0}^{q^{s}} X^{q^{s}}-\mathbf{d}_{n, 1}^{q^{s}} X^{q^{s+1}}+\cdots+(-1)^{n} \mathbf{d}_{n, n}^{q^{s}} X^{q^{n+s}}
$$

has coefficients in $\mathbb{H}$ (cf. Section 5.1 in [3]). Its roots are by construction the linear forms in $\mathbb{F}[V]$. Thus $\mathbb{F}(V)$ is the splitting field of $\Delta(X)$. Hence, the field extension $\mathbb{H} \hookrightarrow \mathbb{F}(V)$ is normal 7 Since $\overline{\mathbb{H}}^{\text {sep }} \hookrightarrow \mathbb{F}(V)$ is purely inseparable, it follows from the structure theorem for finite dimensional normal field extensions that the extension

$$
\mathbb{H} \hookrightarrow \overline{\mathbb{H}}^{s e p}
$$

is Galois with some Galois group $G^{\prime}$. We have

$$
\left|G^{\prime}\right|=\left|\overline{\mathbb{H}}^{s e p}: \mathbb{H}\right|=\left|\mathbb{F}(V): \mathbb{F}(V)^{G}\right|=|G| \text {. }
$$

Since

$$
\mathbb{H}=\left(\overline{\mathbb{H}}^{s e p}\right)^{G^{\prime}}=\overline{\mathbb{H}}^{s e p} \cap \mathbb{F}[V]^{G}
$$

\footnotetext{
${ }^{7}$ Note that this means that $\mathbb{F}(V)$ is algebraically closed in the category of fields over the Steenrod algebra; cf. Section 3.2 in [3].
} 
it follows that $G^{\prime} \geq G$. Thus $G^{\prime}=G$. Finally we show that the $\Delta$-length of an unstable algebra $\mathrm{H}$ coincides with the $\Delta$-length of its separable closure $\left(\overline{\mathrm{H}}^{s e p}\right)$. Together with Lemma 5.1 this gives the result.

To this end, let $l_{i}=\operatorname{dim}_{\mathbb{F}}\left(W_{i}\right)$. Since $G$ acts on $\overline{\mathrm{H}}^{\text {sep }}$, the group $G$ consists of matrices of the form

$$
\left[\begin{array}{ccccc}
A_{0} & 0 & & \ldots & 0 \\
* & A_{1} & 0 & \ldots & 0 \\
& * & \ddots & & \vdots \\
\ldots & & \ddots & & 0 \\
* & & \ldots & * & A_{e}
\end{array}\right]
$$

where $A_{i}$ is an $n_{i} \times n_{i}$-matrix with $n_{i}=\operatorname{dim}\left(W_{i}\right)$. Denote by $\widehat{G}$ the subgroup of $\mathrm{GL}(n, \mathbb{F})$ consisting of all matrices of the form (\#). Denote by $x_{1}, \ldots, x_{n}$ a basis for $W_{0} \oplus \cdots \oplus W_{e}$. Then

$$
\begin{aligned}
\left(\mathbb{F}\left[W_{0}\right]\right. & \left.\otimes \cdots \otimes \mathbb{F}\left[W_{e}\right]^{p^{e}}\right)^{\widehat{G}} \\
& =\mathcal{D}\left(n_{0}\right) \otimes \mathbb{F}\left[c_{\text {top }}\left(x_{n_{0}+1}^{p}\right), \ldots, c_{\text {top }}\left(x_{n_{1}}^{p}\right), c_{\text {top }}\left(x_{n_{1}+1}^{p^{2}}\right), \ldots, c_{\text {top }}\left(x_{n}^{p^{e}}\right),\right.
\end{aligned}
$$

where $c_{\text {top }}(-)$ denotes the top orbit Chern class of the element - (cf. Section 4.1 in [6]). By construction, the top orbit Chern classes of $p$ th powers are $p$ th powers. Thus the $\Delta$-length of the ring of invariants $\widehat{G}$ is equal to $n_{0}$. Therefore we have

$$
\begin{aligned}
\left(\mathbb{F}\left[W_{0}\right] \otimes \cdots \otimes \mathbb{F}\left[W_{e}\right]^{p^{e}}\right)^{\widehat{G}} & \hookrightarrow \mathrm{H}=\left(\mathbb{F}\left[W_{0}\right] \otimes \cdots \otimes \mathbb{F}\left[W_{e}\right]^{p^{e}}\right)^{G} \\
& \hookrightarrow \overline{\mathrm{H}}^{s e p}=\mathbb{F}\left[W_{0}\right] \otimes \cdots \mathbb{F}\left[W_{e}\right]^{p^{e}} .
\end{aligned}
$$

The smallest algebra, as well as the largest algebra in this chain, has $\Delta$-length $n_{0}$. Thus by Lemma 3.7 we are done.

Remark. Since $G$ acts on $\overline{\mathrm{H}}^{\text {sep }}$, the group $G$ consists of matrices of the form given in (\#). So, if there exists no basis such that $G$ consists of flag matrices like above, then the only unstable algebras $\mathrm{H} \hookrightarrow \sqrt[\mathcal{P} *]{\mathrm{H}}=\mathbb{F}[V]^{G}$ are the $p^{s}$ th powers

$$
\mathrm{H}=\left(\mathbb{F}[V]^{G}\right)^{p^{s}}
$$

i.e., we have the trivial vector space decomposition $V=W_{e}$.

Remark. Note carefully that the proof shows that the $\Delta$-length of $\mathrm{H}$ and the $\Delta$ length of any separable extension $\mathrm{H} \hookrightarrow \mathrm{K}$ coincide.

Remark. In Theorem 7.2.2 in [3] as well as in Theorem II in [8] it has been proven that

$$
\mathrm{H}=\left(\mathbb{F}\left[W_{0}\right] \otimes \cdots \otimes \mathbb{F}\left[W_{e}\right]^{p^{e}}\right)^{G}
$$

However, the precise statement on the dimensions of $W_{0} \oplus \cdots \oplus W_{i}$ is missing. Also the connection between the two Galois groups of $\mathrm{H} \hookrightarrow \mathbb{F}\left[W_{0}\right] \otimes \cdots \otimes \mathbb{F}\left[W_{e}\right]^{p^{e}}$, resp. $\sqrt[\mathcal{P}^{*}]{\mathrm{H}} \hookrightarrow \mathbb{F}[V]$, is not made.

We conclude this section with an example.

Example 5.3. Consider the regular representation of the cyclic group of order 2, $\mathbb{Z} / 2$, over a field of characteristic 2 afforded by the matrix

$$
\left[\begin{array}{ll}
1 & 0 \\
1 & 1
\end{array}\right] \text {. }
$$


Set $\mathbb{F}[V]=\mathbb{F}[x, y]$. Its ring of invariants is

$$
\mathbb{F}[x, y]^{\mathbb{Z} / 2}=\mathbb{F}\left[x, y^{2}-y x\right] .
$$

Then $\mathbb{Z} / 2$ acts on $\mathbb{F}\left[x, y^{2}\right]$ with invariant ring

$$
\mathbb{F}\left[x, y^{2}\right]^{\mathbb{Z} / 2}=\mathbb{F}\left[x,\left(y^{2}-y x\right)^{2}\right] .
$$

On the other hand, $\mathbb{Z} / 2$ does not act on $\mathbb{F}\left[x^{2}, y\right]$. Indeed

$$
\mathbb{F}[x, y]^{\mathbb{Z} / 2} \cap \mathbb{F}\left[x^{2}, y\right]=\mathbb{F}\left[x^{2},\left(y^{2}-y x\right)^{2}\right]=\mathbb{F}\left[x^{2}, y^{2}\right]^{\mathbb{Z} / 2} .
$$

Furthermore we could consider the purely inseparable field extension

$$
\mathbb{F}\left(x^{2}, y^{2}-y x\right) \hookrightarrow \mathbb{F}\left(x, y^{2}+x y\right)
$$

of degree 2. Note that

$$
\mathscr{P}^{1}\left(y^{2}+x y\right)=x^{2} y+x y^{2} \notin \mathbb{F}\left(x^{2}, y^{2}-y x\right)
$$

since our field contains only elements of even degree. Thus $\mathbb{F}\left(x^{2}, y^{2}-y x\right)$ is not a field over the Steenrod algebra. Its separable closure

$$
\mathbb{F}\left(x^{2}, y^{2}, y x\right)=\mathbb{F}\left(\frac{x}{y}, y^{2}\right)
$$

is a Galois extension with the same Galois group $\mathbb{Z} / 2$. However, the same calculation as above shows that it is also not closed under the action of the Steenrod algebra, as predicted in the previous result. Indeed, $\mathbb{F}(x, y)$ is the smallest overfield of $\mathbb{F}\left(x^{2}, y^{2}, y x\right)$, say $\mathbb{K}$, closed under the action of the Steenrod algebra as we see next:

$$
\mathscr{P}^{1}(x y)=x^{2} y+x y^{2}=x y(x+y) \in \mathbb{K} \Rightarrow x+y \in \mathbb{K} .
$$

Since $\mathbb{K}$ must have the form $\mathbb{F}(W) \otimes \mathbb{F}(V / W)^{2}$ for some $W \leq V$ we find that $\operatorname{span}_{\mathbb{F}}\{x+y\} \subseteq W$. The minimal polynomial of $x+y \in \mathbb{K}$ over $\mathbb{F}\left(x^{2}, y^{2}, x y\right)$,

$$
p(X)=X^{2}+\left(x^{2}+y^{2}\right),
$$

is inseparable of degree 2. Therefore

$$
2=\mid \mathbb{F}(x, y): \mathbb{F}\left(x^{2}, y^{2}, x y|=| \mathbb{F}(x, y): \mathbb{K}|| \mathbb{K}: \mathbb{F}\left(x^{2}, y^{2}, x y\right)|=2| \mathbb{F}(x, y): \mathbb{K} \mid,\right.
$$

and hence $\mathbb{F}(x, y)=\mathbb{K}$ as claimed.

On the other hand, the largest subfield, call in $\mathbb{L}$, of $\mathbb{F}\left(x^{2}, y^{2}, x y\right)$ that is closed under the Steenrod algebra is $\mathbb{F}\left(x^{2}, y^{2}\right)$ : by Equation (囝) the field $\mathbb{L}$ does not contain $x y$. Since $x y$ is the root of

$$
p(X)=X^{2}+(x y)^{2} \in \mathbb{F}\left(x^{2}, y^{2}\right)[X]
$$

we find that

$$
2=\left|\mathbb{F}\left(x^{2}, y^{2}, x y\right): \mathbb{F}\left(x^{2}, y^{2}\right)\right|=\left|\mathbb{F}\left(x^{2}, y^{2}, x y\right): \mathbb{L}\right|\left|\mathbb{L}: \mathbb{F}\left(x^{2}, y^{2}\right)\right|=2\left|\mathbb{L}: \mathbb{F}\left(x^{2}, y^{2}\right)\right|
$$

and hence $\mathbb{L}=\mathbb{F}\left(x^{2}, y^{2}\right)$. 


\section{Projective dimension}

The goal of this section is to prove that a Noetherian reduced unstable algebra $\mathrm{H}$ is Cohen-Macaulay if and only if its inseparable closure $\sqrt[\mathcal{P} *]{\mathrm{H}}$ is Cohen-Macaulay.

Let $\mathrm{H}$ be an unstable algebra over the Steenrod algebra $\mathcal{P}^{*}$. An ideal $I \subseteq \mathrm{H}$ is called $\mathcal{P}^{*}$-invariant if it is closed under the action of the Steenrod algebra.

Lemma 6.1. Let $\mathrm{H}$ be an unstable algebra over the Steenrod algebra. For any $s \in \mathbb{N}_{0}$, the canonical inclusion

$$
\psi: \mathrm{H}^{p^{s}} \hookrightarrow \mathrm{H}
$$

induces a bijection

$$
\psi^{*}: \mathcal{P}_{\operatorname{roj}} j_{\mathcal{P} *}(\mathrm{H}) \rightarrow \mathcal{P} \operatorname{roj}_{\mathcal{P} *}\left(\mathrm{H}^{p^{s}}\right)
$$

between the spaces of homogeneous $\mathcal{P}^{*}$-invariant prime ideals.

Proof. Since $\psi$ is an integral extension, the Lying-Over Theorem holds. Thus $\psi^{*}$ is surjective.

To prove injectivity take two homogeneous $\mathcal{P}^{*}$-invariant prime ideals $\mathfrak{p}_{1}, \mathfrak{p}_{2} \subseteq \mathrm{H}$, such that

Thus for any $h \in \mathfrak{p}_{1}$ it follows that

$$
\mathfrak{p}_{1} \cap \mathrm{H}^{p^{s}}=\mathfrak{p}_{2} \cap \mathrm{H}^{p^{s}} .
$$

$$
h^{p^{s}} \in \mathfrak{p}_{1} \cap \mathrm{H}^{p^{s}}=\mathfrak{p}_{2} \cap \mathrm{H}^{p^{s}} .
$$

Therefore

$$
h^{p^{s}} \in\left(\psi\left(\mathfrak{p}_{2} \cap \mathrm{H}^{p^{s}}\right)\right) \subseteq \mathfrak{p}_{2} .
$$

Since $\mathfrak{p}_{2}$ is prime, we find that $h \in \mathfrak{p}_{2}$. Interchanging the roles of $\mathfrak{p}_{1}$ and $\mathfrak{p}_{2}$ gives the result.

This result could have been proven also by observing that the sth iteration of the Frobenius map

$$
F^{s}: \mathrm{H} \rightarrow \mathrm{H}^{p^{s}}
$$

hands us an isomorphism of unstable algebras of degree $p^{s}$ if $\mathrm{H}$ is reduced. This in turn also implies the following result.

Lemma 6.2. Let $\mathrm{H}$ be an unstable reduced algebra over the Steenrod algebra. For any $s \in \mathrm{N}_{0}$ we find that

$$
\operatorname{depth}(\mathrm{H})=\operatorname{depth}\left(\mathrm{H}^{p^{s}}\right)
$$

We observe that $\mathrm{H}$ is Noetherian of Krull dimension $n$ if and only if $\mathrm{H}^{p^{s}}$ is. We find the following lemma.

Lemma 6.3. Let $\mathrm{H}$ be Noetherian and reduced of Krull dimension n. Let $S=$ $\mathbb{F}\left[h_{1}^{p^{s}}, \ldots, h_{n}^{p^{s}}\right]$ be a system of parameters in $\mathrm{H}^{p^{s}}$. Then

$$
\operatorname{proj}-\operatorname{dim}_{S}(\mathrm{H})=\operatorname{proj}-\operatorname{dim}_{S}\left(\mathrm{H}^{p^{s}}\right)<\infty .
$$

Proof. Since $\mathrm{H}^{p^{s}} \subseteq \mathrm{H}$ is a finite integral extension, $S$ is also a system of parameters for $\mathrm{H}$. Thus both projective dimensions are finite. Moreover, by the AuslanderBuchsbaum formula we have

$\operatorname{proj}-\operatorname{dim}_{S}\left(\mathrm{H}^{p^{s}}\right)=\operatorname{dim}\left(\mathrm{H}^{p^{s}}\right)-\operatorname{depth}\left(\mathrm{H}^{p^{s}}\right)=\operatorname{dim}(\mathrm{H})-\operatorname{depth}(\mathrm{H})=\operatorname{proj}-\operatorname{dim}_{S}(\mathrm{H})$ by Lemma 6.2. 
We come to the desired result about a Noetherian unstable algebra $\mathrm{H}$ and its $\mathcal{P}^{*}$-inseparable closure $\sqrt[\mathcal{P}^{*}]{\mathrm{H}}$.

Proposition 6.4. Let $\mathrm{H}$ be Noetherian and reduced of Krull dimension n. Then $\mathrm{H}$ is Cohen-Macaulay if and only if $\sqrt[\mathcal{P *}]{\mathrm{H}}$ is Cohen-Macaulay.

Proof. Since H is Noetherian, its $\mathcal{P}^{*}$-inseparable closure is also Noetherian by Theorem 6.1.3 in [3]. Therefore $\sqrt[\mathcal{P}^{*}]{\mathrm{H}}=\mathrm{H}_{s}$ for some $s \in \mathbb{N}_{0}$. Thus

$$
(\sqrt[\mathcal{P} *]{\mathrm{H}})^{p^{s}} \hookrightarrow \mathrm{H} \hookrightarrow \sqrt[\mathcal{P}^{*}]{\mathrm{H}}=\mathrm{H}_{s}
$$

is a finite integral extension. By Lemma 6.1 we have a bijection

$$
\operatorname{Proj}_{\mathcal{P} *}\left(\sqrt[\mathcal{P}^{*}]{\mathrm{H}}\right)^{p^{s}} \rightarrow \mathcal{P} \operatorname{roj}_{\mathcal{P}^{*}}\left(\sqrt[\mathcal{P}^{*}]{\mathrm{H}}\right)
$$

By Theorem 4.3.1 in [3] and Lemma 6.1

$$
\operatorname{Proj}_{\mathcal{P}^{*}}\left(\sqrt[\mathcal{P}^{*}]{\mathrm{H}}\right)^{p^{s}} \rightarrow \mathcal{P} \operatorname{joj}_{\mathcal{P}^{*}}(\mathrm{H}) \rightarrow \mathcal{P}_{\operatorname{roj}} j_{\mathcal{P}^{*}}\left(\sqrt[\mathcal{P}^{*}]{\mathrm{H}}\right)
$$

is also bijective. Moreover, by Lemma 6.2 the left and the right algebra have the same depth. Thus by Theorem 2.1 in 5 the results follows (cf. Corollary 2.2 loc.cit.).

\section{Polynomial Rings}

Let $\mathrm{H}$ be an integrally closed unstable Noetherian integral domain over the Steenrod algebra. By Theorem 5.2 we have

$$
\mathrm{H}=\left(\overline{\mathrm{H}}^{s e p}\right)^{G} \hookrightarrow \sqrt[\mathcal{P}^{*}]{\mathrm{H}}=\mathbb{F}[V]^{G},
$$

where

$$
\overline{\mathrm{H}}^{s e p}=\mathbb{F}\left[W_{0}\right] \otimes \cdots \otimes \mathbb{F}\left[W_{e}\right]^{p^{e}}
$$

for some vector space decomposition $V=W_{0} \oplus \cdots \oplus W_{e}$. By Proposition 6.4 we know that $\mathrm{H}$ is Cohen-Macaulay if and only if $\sqrt[\mathcal{P}^{*}]{\mathrm{H}}$ is polynomial. Moreover, the algebra generators of $\mathrm{H}$ are just suitable $p^{s}$ th powers of the algebra generators of $\sqrt[\mathcal{P}^{*}]{\mathrm{H}}$ (for a minimal generating set).

Let $G$ act on $V=W_{0} \oplus \cdots \oplus W_{e}$ such that

$$
g w_{i} \in W_{0} \oplus \cdots \oplus W_{i}
$$

for all $w_{i} \in W_{i}$, i.e., $G$ consists of flag matrices of the form

$$
\left[\begin{array}{ccccc}
A_{0} & 0 & & \ldots & 0 \\
* & A_{1} & 0 & \ldots & 0 \\
& * & \ddots & & \vdots \\
\cdots & & \ddots & & 0 \\
* & & \ldots & * & A_{e}
\end{array}\right]
$$

where $A_{i}$ is an $m_{i} \times m_{i}$-matrix with $m_{i}=\operatorname{dim}\left(W_{i}\right)$. For every $i=0, \ldots, e$ we have a group epimorphism

$$
\operatorname{pr}_{i}: G \rightarrow G_{i},\left[\begin{array}{ccccc}
A_{0} & 0 & & \ldots & 0 \\
* & A_{1} & 0 & \ldots & 0 \\
& * & \ddots & & \vdots \\
\ldots & & \ddots & & 0 \\
* & & \ldots & * & A_{e}
\end{array}\right] \mapsto\left[\begin{array}{ccccc}
A_{0} & 0 & & \ldots & 0 \\
* & A_{1} & 0 & \ldots & 0 \\
& * & \ddots & & \vdots \\
\ldots & & \ddots & & 0 \\
* & & \ldots & * & A_{i}
\end{array}\right] .
$$


Lemma 7.1. With the preceding notation we have

$$
\mathbb{F}\left[W_{0} \oplus \cdots \oplus W_{i}\right]^{G_{i}}=\mathbb{F}[V]^{G} \cap \mathbb{F}\left[W_{0} \oplus \cdots \oplus W_{i}\right] \subseteq \mathbb{F}[V]^{G} .
$$

Proof. The kernel of the projection $\operatorname{pr}_{i}, \operatorname{ker}\left(\operatorname{pr}_{i}\right)$, consists of matrices of the form

$$
\left[\begin{array}{cccccc}
I_{0} & 0 & & & \ldots & 0 \\
0 & \ddots & & & & 0 \\
\vdots & & I_{i} & 0 & \ldots & 0 \\
* & * & * & A_{i+1} & & \vdots \\
& & & * & \ddots & \\
* & & \ldots & * & & A_{e}
\end{array}\right],
$$

where the $I_{j}$ 's are identity matrices. Thus $\mathbb{F}[V]^{\operatorname{ker}\left(\mathrm{pr}_{i}\right)} \supseteq \mathbb{F}\left[W_{0} \oplus \cdots \oplus W_{i}\right]$, and hence

$$
\mathbb{F}[V]^{G}=\left(\mathbb{F}[V]^{\operatorname{ker}\left(\mathrm{pr}_{i}\right)}\right)^{G_{i}} \supseteq \mathbb{F}\left[W_{0} \oplus \cdots \oplus W_{i}\right]^{G_{i}} .
$$

Since $\mathbb{F}\left[W_{0} \oplus \cdots \oplus W_{i}\right]^{G_{i}} \subseteq \mathbb{F}\left[W_{0} \oplus \cdots \oplus W_{i}\right]$ we find

$$
\mathbb{F}\left[W_{0} \oplus \cdots \oplus W_{i}\right]^{G_{i}} \subseteq \mathbb{F}[V]^{G} \cap \mathbb{F}\left[W_{0} \oplus \cdots \oplus W_{i}\right] \subseteq \mathbb{F}[V]^{G} .
$$

Conversely, since $\mathbb{F}[V]^{\operatorname{ker}\left(\mathrm{pr}_{i}\right)} \supseteq \mathbb{F}\left[W_{0} \oplus \cdots \oplus W_{i}\right]$, we have

$$
\mathbb{F}[V]^{\operatorname{ker}\left(\mathrm{pr}_{i}\right)} \cap \mathbb{F}\left[W_{0} \oplus \cdots \oplus W_{i}\right]=\mathbb{F}\left[W_{0} \oplus \cdots \oplus W_{i}\right] .
$$

Thus

$$
\mathbb{F}\left[W_{0} \oplus \cdots \oplus W_{i}\right]^{G_{i}}=\left(\mathbb{F}[V]^{\operatorname{ker}\left(\operatorname{pr}_{i}\right)} \cap \mathbb{F}\left[W_{0} \oplus \cdots \oplus W_{i}\right]\right)^{G_{i}} .
$$

Finally, note that

$$
\mathbb{F}[V]^{G} \cap \mathbb{F}\left[W_{0} \oplus \cdots \oplus W_{i}\right] \subseteq\left(\mathbb{F}[V]^{\operatorname{ker}\left(\operatorname{pr}_{i}\right)} \cap \mathbb{F}\left[W_{0} \oplus \cdots \oplus W_{i}\right]\right)^{G_{i}} .
$$

To see this, take an element $f \in \mathbb{F}[V]^{G} \cap \mathbb{F}\left[W_{0} \oplus \cdots \oplus W_{i}\right]$. Then $f \in \mathbb{F}\left[W_{0} \oplus \cdots \oplus W_{i}\right]$ is invariant under the group $G$. Thus $f$ is also invariant under $\operatorname{ker}\left(\operatorname{pr}_{i}\right)$. Therefore,

$$
f \in \mathbb{F}[V]^{\operatorname{ker}\left(\operatorname{pr}_{i}\right)} \cap \mathbb{F}\left[W_{0} \oplus \cdots \oplus W_{i}\right] .
$$

But $f$ is also $G$-invariant, i.e.,

$$
f \in\left(\mathbb{F}[V]^{\operatorname{ker}\left(\mathrm{pr}_{i}\right)} \cap \mathbb{F}\left[W_{0} \oplus \cdots \oplus W_{i}\right]\right)^{G} \subseteq\left(\mathbb{F}[V]^{\operatorname{ker}\left(\operatorname{pr}_{i}\right)} \cap \mathbb{F}\left[W_{0} \oplus \cdots \oplus w_{i}\right]\right)^{G_{i}}
$$

as desired.

Let $h_{1}, \ldots, h_{m} \in \mathbb{F}[V]^{G}$ be a minimal generating set. Without loss of generality we assume that they are sorted such that

$$
\begin{aligned}
& h_{1}, \ldots, h_{n_{0}} \in \mathbb{F}\left[W_{0}\right], \\
& h_{n_{0}+1}, \ldots, h_{n_{1}} \in \mathbb{F}\left[W_{0} \oplus W_{1}\right], \\
& \ldots \\
& h_{n_{e-1}+1}, \ldots, h_{n_{e}}=h_{m} \in \mathbb{F}\left[W_{0} \oplus \cdots \oplus W_{e}\right] .
\end{aligned}
$$

We assume that $n_{0}, \ldots, n_{e}$ are maximal with this property. Thus by construction

$$
\mathbb{F}\left[h_{0}, \ldots, h_{n_{i}}\right] \subseteq \mathbb{F}\left[W_{0} \oplus \cdots \oplus W_{i}\right]^{G_{i}} \subseteq \mathbb{F}[V]^{G}
$$

for all $i=0, \ldots, e$. 
Proposition 7.2. If $n_{e}=\operatorname{dim}_{\mathbb{F}}(V)$, i.e., if the ring of invariants

$$
\mathbb{F}\left[W_{0} \oplus \cdots \oplus W_{e}\right]^{G}
$$

is polynomial, then $n_{i}=\operatorname{dim}_{\mathbb{F}}\left(W_{0} \oplus \cdots \oplus W_{i}\right)$.

Proof. Consider the integral extension

$$
\mathbb{F}\left[W_{0} \oplus \cdots \oplus W_{i}\right]^{G_{i}} \hookrightarrow \mathbb{F}\left[W_{0} \oplus \cdots \oplus W_{i}\right] .
$$

The maximal ideal $\mathfrak{m}_{i}$ of $\mathbb{F}\left[W_{0} \oplus \cdots \oplus W_{i}\right]$ lies over the maximal ideal in $\mathbb{F}\left[W_{0} \oplus\right.$ $\left.\cdots \oplus W_{i}\right]^{G_{i}}$. Furthermore, $\mathfrak{m}_{i}$ extends to a prime ideal $\mathfrak{p}_{i} \subseteq \mathbb{F}\left[W_{0} \oplus \cdots \oplus W_{e}\right]$. By construction $\mathfrak{p}_{i}$ is generated by all linear forms in $\mathbb{F}\left[W_{0} \oplus \cdots \oplus W_{i}\right]$. Thus $\mathfrak{p}_{i}$ is regular and prime of height equal to $\operatorname{dim}_{\mathbb{F}}\left(W_{0} \oplus \cdots \oplus W_{i}\right)$. Hence, its contraction to the ring of invariants

$$
\mathfrak{p}_{i}^{c}=\mathfrak{p}_{i} \cap \mathbb{F}\left[W_{0} \oplus \cdots \oplus W_{e}\right]^{G}
$$

is also prime of height equal to $\operatorname{dim}_{\mathbb{F}}\left(W_{0} \oplus \cdots \oplus W_{i}\right)$. Furthermore, $\mathfrak{p}_{i}^{c}$ contains by construction

$$
\left(h_{1}, \ldots, h_{n_{i}}\right) \subseteq \mathfrak{p}_{i}^{c} .
$$

Thus the quotient

$$
\mathbb{F}\left[W_{0} \oplus \cdots \oplus W_{e}\right]^{G} / \mathfrak{p}^{c}=\mathbb{F}\left[\bar{h}_{n_{i}+1}, \ldots, \bar{h}_{n}\right] \hookrightarrow \mathbb{F}\left[W_{i+1} \oplus \cdots \oplus W_{e}\right]
$$

is integral, and

$$
n-n_{i}=\operatorname{dim}_{\mathbb{F}}\left(W_{i+1} \oplus \cdots \oplus W_{e}\right)
$$

for all $i=0, \ldots, e-1$.

Theorem 7.3. With the above notation, if

$$
\left(\mathbb{F}\left[W_{0}\right] \otimes \cdots \otimes \mathbb{F}\left[W_{e}\right]^{p^{e}}\right)^{G}=\mathbb{F}\left[h_{1}, \ldots, h_{n}\right]
$$

is polynomial, then for suitable $s_{1}, \ldots, s_{n} \in \mathbb{N}_{0}$

$$
\left(\mathbb{F}\left[U_{0}\right] \otimes \cdots \otimes \mathbb{F}\left[U_{f}\right]^{p^{f}}\right)^{G}=\mathbb{F}\left[h_{1}^{p^{s_{1}}}, \ldots, h_{n}^{p^{s_{n}}}\right]
$$

is polynomial for any subflag

$$
U_{0} \oplus \cdots \oplus U_{j} \leq W_{0} \oplus \cdots \oplus W_{i}
$$

that admits an action of $G$.

Proof. To simplify notation we assume that the extension

$$
\mathbb{F}\left[U_{0}\right] \otimes \cdots \otimes \mathbb{F}\left[U_{f}\right]^{p^{f}} \hookrightarrow \mathbb{F}\left[W_{0}\right] \otimes \cdots \otimes \mathbb{F}\left[W_{e}\right]^{p^{e}}
$$

is purely inseparable of exponent one. The general case follows then inductively.

Since $G$ acts on the flag $W_{0} \oplus \cdots \oplus W_{e}$ the algebra generator for the ring of invariants can be sorted such that

$$
h_{1}, \ldots, h_{n_{i}} \in \mathbb{F}\left[W_{0} \oplus \cdots \oplus W_{i}\right]
$$

with $n_{i}-n_{i-1}=\operatorname{dim}\left(W_{i}\right), n_{0}=\operatorname{dim}_{\mathbb{F}}\left(W_{0}\right)$, by Proposition 7.2 ,

Since $G$ acts also on the subflag $U_{0} \oplus \cdots \oplus U_{f}$ the algebra generator for the ring of invariants can be sorted such that

$$
h_{1}, \ldots, h_{m_{i}} \in \mathbb{F}\left[U_{0} \oplus \cdots \oplus U_{i}\right]
$$


with $m_{i}-m_{i-1}=\operatorname{dim}\left(U_{i}\right), m_{0}=\operatorname{dim}_{\mathbb{F}}\left(U_{0}\right)$, and $m_{f}=n_{e}=n$. Thus $n_{i} \geq m_{i}$. Consider the algebra

$$
\begin{aligned}
\mathrm{A}= & \mathbb{F}\left[h_{1}, \ldots, h_{m_{0}}, h_{m_{0}+1}^{p}, \ldots, h_{n_{0}}^{p}, h_{n_{0}+1}, \ldots,\right. \\
& \left.h_{m_{1}}, h_{m_{1}+1}^{p}, \ldots, h_{n_{1}}^{p}, \ldots, h_{m_{e}}, h_{m_{e}+1}^{p}, \ldots, h_{m_{f}}^{p}\right] \\
\hookrightarrow & \mathbb{F}\left[U_{0}\right] \otimes \cdots \otimes \mathbb{F}\left[U_{f}\right]^{p^{f}} .
\end{aligned}
$$

Since A consists of invariant polynomials it is contained in the ring of invariants

$$
\left(\mathbb{F}\left[U_{0}\right] \otimes \cdots \otimes \mathbb{F}\left[U_{f}\right]^{p^{f}}\right)^{G} .
$$

The diagram

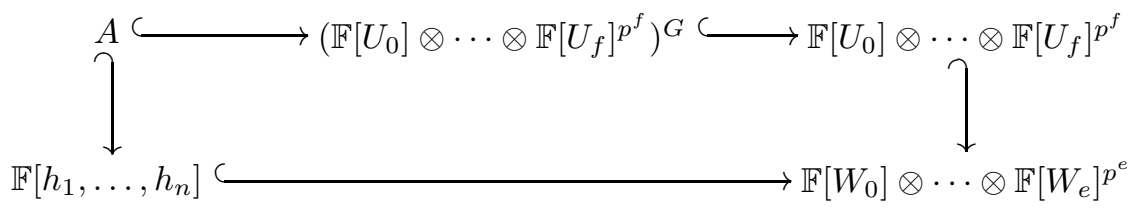

has by construction purely inseparable vertical extensions of degree $p^{\sum_{i}\left(n_{i}-m_{i}\right)}$. Since the degree of

$$
\mathbb{F}\left[h_{1}, \ldots, h_{n}\right] \hookrightarrow \mathbb{F}\left[W_{0}\right] \otimes \cdots \otimes \mathbb{F}\left[W_{e}\right]^{p^{e}}
$$

is the group order $|G|$, the degree of

$$
A \hookrightarrow \mathbb{F}\left[U_{0}\right] \otimes \cdots \otimes \mathbb{F}\left[U_{f}\right]^{p^{f}}
$$

is also the group order. Thus A is the desired ring of invariants as claimed.

The following result settles a twenty-year-old conjecture due to Clarence W. Wilkerson (see Conjecture 5.1 in [8]).

Theorem 7.4. Let $\mathrm{H}$ be an integrally closed Noetherian unstable integral domain over the Steenrod algebra. Then $\mathrm{H}$ is polynomial if and only if $\sqrt[\mathcal{P}^{*}]{\mathrm{H}}$ is polynomial. Furthermore,

$$
\sqrt[\mathcal{P}^{*}]{\mathrm{H}}=\mathbb{F}\left[h_{1}, \ldots, h_{n}\right]
$$

if and only if there are $s_{1}, \ldots, s_{n} \in \mathbb{N}_{0}$ such that

$$
\mathrm{H}=\mathbb{F}\left[h_{1}^{p^{s_{1}}}, \ldots, h_{n}^{p^{n}}\right] .
$$

Proof. By Theorem 5.2 there exist a group $G$ and a flag $V=W_{0} \oplus \cdots \oplus W_{e}$ such that

$$
\mathrm{H}=\left(\mathbb{F}\left[W_{0}\right] \otimes \cdots \otimes \mathbb{F}\left[W_{e}\right]^{p^{e}}\right)^{G} \hookrightarrow \sqrt[\mathcal{P}^{*}]{\mathrm{H}}=\mathbb{F}[V]^{G} .
$$

If $\sqrt[P^{*}]{\mathrm{H}}$ is polynomial, then so is $\mathrm{H}$ by Theorem 7.3 , Note that the same result also gives the precise statement on the respective algebra generators.

On the other hand, $\left(\sqrt[\mathcal{P}^{*}]{\mathrm{H}}\right)^{p^{e}}=\left(\mathbb{F}[V]^{p^{e}}\right)^{G} \hookrightarrow \mathrm{H}$ is the ring of invariants on the subflag $V \leq W_{0} \oplus \cdots \oplus W_{e}$ for some large enough $e$. Therefore if $\mathrm{H}$ is polynomial, then $\left(\sqrt[\mathcal{P}^{*}]{\mathrm{H}}\right)^{p^{e}} \hookrightarrow \mathrm{H}$ is polynomial by the same Theorem 7.3 Thus $\sqrt[\mathcal{P}^{*}]{\mathrm{H}}$ is polynomial since it is isomorphic as an algebra to $(\sqrt[\mathcal{P} *]{\mathrm{H}})^{p^{e}}$.

Thus we have the following corollary.

Corollary 7.5. Let $\mathrm{H}$ be an unstable polynomial algebra over the Steenrod algebra. Set $\mathrm{H}=\mathbb{F}\left[h_{1}, \ldots, h_{n}\right]$. Then $\mathrm{H}$ is $\mathcal{P}^{*}$-inseparably closed if and only if the polynomial generators $h_{1}, \ldots, h_{n}$ are no pth powers. 
The example given at the end of Section 5 illustrates these results. We want to close with an example that shows that a simple generalization of Theorem 7.4 to nonpolynomial invariants is not true.

Example 7.6. Let $p$ be odd and let $\mathbb{F}$ be the prime field of characteristic $p$. Consider the four-dimensional modular representation $\mathbb{Z} / p \hookrightarrow \mathrm{GL}(4, \mathbb{F})$ afforded by the matrix

$$
\left[\begin{array}{llll}
1 & 1 & 0 & 0 \\
0 & 1 & 0 & 0 \\
0 & 0 & 1 & 1 \\
0 & 0 & 0 & 1
\end{array}\right] .
$$

Its ring of invariants turns out to be a hypersurface

$$
\mathbb{F}\left[x_{1}, y_{1}, x_{2}, y_{2}\right]^{\mathbb{Z} / p}=\mathbb{F}\left[c_{1}, y_{1}, c_{2}, y_{2}, q\right] /(r),
$$

where $c_{i}=x_{i}^{p}-x_{i} y_{i}^{p-1}$ are the top orbit Chern classes of $x_{i}, i=1,2$, and $q=$ $x_{1} y_{2}-x_{1} y_{1}$ is an invariant quadratic form. The relation is given by

$$
r=q^{p}-c_{1} y_{2}^{p}+c_{2} y_{1}^{p}+q y_{1}^{p-1} y_{2}^{p-1}
$$

(see Theorem 2.1 in [2]). Certainly, $\mathbb{Z} / p$ also acts on $\mathbb{F}\left[x_{1}, y_{1}\right] \otimes \mathbb{F}\left[x_{2}^{p}, y_{2}^{p}\right]$ and we find that

$$
\mathrm{A}=\mathbb{F}\left[c_{1}, y_{1}, c_{2}^{p}, y_{2}^{p}, q^{p}\right] \hookrightarrow\left(\mathbb{F}\left[x_{1}, y_{1}\right] \otimes \mathbb{F}\left[x_{2}^{p}, y_{2}^{p}\right]\right)^{\mathbb{Z} / p} .
$$

However, the new ring of invariants contains an invariant that is not in the algebra A, namely

$$
q^{\prime}=x_{1} y_{2}^{p}-x_{2}^{p} y_{1} .
$$

Indeed, with the methods presented in Theorem 2.1 of [2] it is not hard to see that

$$
\left(\mathbb{F}\left[x_{1}, y_{1}\right] \otimes \mathbb{F}\left[x_{2}^{p}, y 2^{p}\right]\right)^{\mathbb{Z} / p}=\mathbb{F}\left[c_{1}, y_{1}, c_{2}^{p}, y_{2}^{p}, q^{\prime}\right] /\left(r^{\prime}\right),
$$

where $r^{\prime}=\left(q^{\prime}\right)^{p}-c_{1} y_{2}^{p^{2}}+c_{2}^{p} y_{1}^{p}-q^{\prime} y_{1}^{p-1} y_{2}^{p(p-1)}$. Interesting enough though, it transpires that this ring is again a hypersurface.

\section{REFERENCES}

[1] Nathan Jacobson, Lecture in Abstract Algebra, Vol. III: Theory of Fields and Galois Theory, D. van Nostrand Company, Inc., Princeton, NJ, 1964. MR0172871 (30:3087)

[2] Mara D. Neusel, Invariants of some Abelian p-Groups in Characteristic p, Proc. Amer. Math. Soc. 125 (1997), 1921-1931. MR1377000 (97i:13004)

[3] Mara D. Neusel, Inverse Invariant Theory and Steenrod Operations, Memoirs Amer. Math. Soc., vol. 146, AMS, Providence, RI, 2000. MR1693799 (2000m:55023)

[4] Mara D. Neusel, Localizations over the Steenrod Algebra. The lost Chapter, Math. Zeitschrift 235 (2000), 353-378. MR.1795513 (2001m:16033)

[5] Mara D. Neusel, Unstable Cohen-Macaulay Algebras, Mathematical Research Letters 8 (2001), 1-13. MR1839483 (2002d:13030)

[6] Mara D. Neusel and Larry Smith, Invariant Theory of Finite Groups, Mathematical Surveys and Monographs 94, AMS, Providence, RI, 2002. MR.1869812 (2002k:13012)

[7] Clarence W. Wilkerson, Classifying Spaces, Steenrod Operations and Algebraic Closure, Topology 16 (1977), 227-337. MR0442932 (56:1307)

[8] Clarence W. Wilkerson, Rings of Invariants and Inseparable Forms of Algebras over the Steenrod Algebra, pp. 381-396 in: Recent progress in homotopy theory (Baltimore, MD, 2001), Contemp. Math. 293, AMS, Providence, RI, 2002. MR.1890745 (2003d:55020)

Department of Mathematics and Statistics, Texas Tech University, Lubbock, Texas 79409-1042

E-mail address: Mara.D.Neusel@ttu.edu 